Pacific

Journal of

Mathematics

EFFECTIVE DIVISORS ON THE PROJECTIVE LINE HAVING SMALL DIAGONALS AND SMALL HEIGHTS AND THEIR APPLICATION TO ADELIC DYNAMICS

YÛSUKE OKUYAMA 


\title{
EFFECTIVE DIVISORS ON THE PROJECTIVE LINE HAVING SMALL DIAGONALS AND SMALL HEIGHTS AND THEIR APPLICATION TO ADELIC DYNAMICS
}

\author{
YÛSUKE OKUYAMA
}

\begin{abstract}
We establish a quantitative adelic equidistribution theorem for a sequence of effective divisors on the projective line over the separable closure of a product formula field having small diagonals and small $g$-heights with respect to an adelic normalized weight $g$ in arbitrary characteristic and in a possibly nonseparable setting. Applying this quantitative adelic equidistribution result to adelic dynamics of $f$, we obtain local proximity estimates between the iterations of a rational function $f \in k(z)$ of degree $>1$ and a rational function $a \in k(z)$ of degree $>0$ over a product formula field $k$ of characteristic 0 .
\end{abstract}

\section{Introduction}

Let $k$ be a field and denote by $k_{s}$ the separable closure of $k$ in an algebraic closure $\bar{k}$. For every $d \in \mathbb{N} \cup\{0\}$, let $k\left[p_{0}, p_{1}\right]_{d}$ be the set of all homogeneous polynomials in two variables over $k$ of degree $d$. A $k$-effective divisor $\mathcal{Z}$ on $\mathbb{P}^{1}(\bar{k})$ is a divisor on $\mathbb{P}^{1}(\bar{k})$ defined by the zeros in $\mathbb{P}^{1}(\bar{k})$ of some $P \in \bigcup_{d \in \mathbb{N}} k\left[p_{0}, p_{1}\right]_{d}$ taking into account their multiplicities, and is said to be on $\mathbb{P}^{1}\left(k_{s}\right)$ if $\operatorname{supp} \mathcal{Z} \subset \mathbb{P}^{1}\left(k_{s}\right)$. The defining polynomial $P\left(p_{0}, p_{1}\right)$ of $\mathcal{Z}$ is unique up to multiplication in $k^{*}(=k \backslash\{0\})$, and is called a representative of $\mathcal{Z}$. Effective divisors include Galois conjugacy classes of algebraic numbers, and are also called Galois stable multisets in $\mathbb{P}^{1}(\bar{k})$.

Our first aim in this article is to establish a quantitative adelic equidistribution of sequences of $k$-effective divisors on $\mathbb{P}^{1}\left(k_{s}\right)$, where $k$ is a product formula field, having not only small $g$-heights (with respect to an adelic normalized weight $g$ ) but also small diagonals in arbitrary characteristic and in a possibly nonseparable setting. Secondly, we contribute to the study of the local proximities between the iterations of a rational function $f \in k(z)$ of degree $>1$ and a rational function $a \in k(z)$ of degree $>0$ on a chordal disk $D$ of radius $>0$ in the projective line $\mathbb{P}^{1}\left(\mathbb{C}_{v}\right)$ for each place $v$ of $k$, in the setting of adelic dynamics of characteristic 0.

MSC2010: primary 37P30; secondary 11G50, 37P50, 37F10.

Keywords: product formula field, effective divisor, small diagonals, small heights, quantitative equidistribution, asymptotically Fekete configuration, local proximity sequence, adelic dynamics. 
1.1. Arithmetic over a product formula field. A field $k$ is a product formula field if $k$ is equipped with

(i) a set $M_{k}$ of all places of $k$, which are either finite or infinite,

(ii) a set $\left\{|\cdot|_{v}: v \in M_{k}\right\}$, where for each $v \in M_{k},|\cdot|_{v}$ is a nontrivial absolute value of $k$ representing $v$ (and then by definition $|\cdot|_{v}$ is nonarchimedean if and only if $v$ is finite), and

(iii) a set $\left\{N_{v}: v \in M_{k}\right\}$, where $N_{v} \in \mathbb{N}$ for every $v \in M_{k}$

such that the following product formula holds: if $z \in k \backslash\{0\}$ then we have $|z|_{v} \neq 1$ for at most finitely many $v \in M_{k}$ and moreover

$$
\prod_{v \in M_{k}}|z|_{v}^{N_{v}}=1 .
$$

Product formula fields include number fields and function fields over curves, and a product formula field is a number field if and only if it has at least one infinite place (see, e.g., the paragraph after Definition 7.51 of [Baker and Rumely 2010]).

Let $k$ be a product formula field. For each $v \in M_{k}$, let $k_{v}$ be the completion of $k$ with respect to $|\cdot|_{v}$ and $\mathbb{C}_{v}$ the completion of an algebraic closure of $k_{v}$ with respect to (the extended) $|\cdot|_{v}$. We fix an embedding of $\bar{k}$ into $\mathbb{C}_{v}$ which extends that of $k$ into $k_{v}$; by convention, the dependence on $v \in M_{k}$ of a local quantity induced by $|\cdot|_{v}$ is emphasized by adding the suffix $v$ to it. A family $g=\left\{g_{v}: v \in M_{k}\right\}$ is an adelic continuous weight if

(i) for every $v \in M_{k}, g_{v}$ is a continuous function on the Berkovich projective line $\mathrm{P}^{1}\left(\mathbb{C}_{v}\right)$ such that

$$
\mu_{v}^{g}:=\Delta g_{v}+\Omega_{\mathrm{can}, v}
$$

is a probability Radon measure on $\mathrm{P}^{1}\left(\mathbb{C}_{v}\right)$ (see (2-2) for the definition of the probability Radon measure $\Omega_{\text {can, } v}$ on $\mathrm{P}^{1}\left(\mathbb{C}_{v}\right)$, and (2-3) for the normalization of the Laplacian $\Delta$ on $\mathrm{P}^{1}\left(\mathbb{C}_{v}\right)$ ), and

(ii) there is a finite subset $E_{g}$ in $M_{k}$ such that $g_{v} \equiv 0$ on $\mathrm{P}^{1}\left(\mathbb{C}_{v}\right)$ for all $v \in M_{k} \backslash E_{g}$. Moreover, $g$ is called an adelic normalized weight if, in addition,

(iii) the $g_{v}$-equilibrium energy $V_{g_{v}}$ of $\mathrm{P}^{1}\left(\mathbb{C}_{v}\right)$ vanishes for every $v \in M_{k}$ (see Section 2.1 for the definition of $V_{g_{v}}$ ).

For an adelic continuous weight $g=\left\{g_{v}: v \in M_{k}\right\}$, the family $\mu^{g}:=\left\{\mu_{v}^{g}: v \in M_{k}\right\}$ is called an adelic probability measure (compare [Favre and Rivera-Letelier 2006, Définition 1.1]). An adelic continuous weight $g=\left\{g_{v}: v \in M_{k}\right\}$ is said to be placewise Hölder continuous if for every $v \in M_{k}, g_{v}$ is Hölder continuous on $\mathrm{P}^{1}\left(\mathbb{C}_{v}\right)$ with respect to the small model metric $\mathrm{d}_{v}$ on $\mathrm{P}^{1}\left(\mathbb{C}_{v}\right)$ (see (3-1) for the definition of $\mathrm{d}_{v}$ ). 
Given $P \in \bigcup_{d \in \mathbb{N}} k\left[p_{0}, p_{1}\right]_{d}$ and an adelic continuous weight $g=\left\{g_{v}: v \in M_{k}\right\}$, the $g$-height of a $k$-effective divisor $\mathcal{Z}$ on $\mathbb{P}^{1}(\bar{k})$ represented by $P$ is

$$
h_{g}(\mathcal{Z}):=\sum_{v \in M_{k}} N_{v} \frac{M_{g_{v}}(P)}{\operatorname{deg} P},
$$

where, for every $v \in M_{k}, M_{g_{v}}(P)$ is the logarithmic $g_{v}$-Mahler measure of $P$ (see (2-10) for the definition of $M_{g_{v}}(P)$ and Section 2.3 for a proof that $\left.h_{g}(\mathcal{Z}) \in \mathbb{R}\right)$; by $(\mathrm{PF}), h_{g}(\mathcal{Z})$ is well defined. For every $v \in M_{k}$, letting $\delta_{\mathcal{S}}$ be the Dirac measure on $\mathrm{P}^{1}\left(\mathbb{C}_{v}\right)$ at a point $\mathcal{S} \in \mathrm{P}^{1}\left(\mathbb{C}_{v}\right)$, a $k$-effective divisor $\mathcal{Z}$ on $\mathbb{P}^{1}(\bar{k})$ is regarded as a positive and discrete Radon measure $\sum_{w \in \operatorname{supp} \mathcal{Z}}\left(\operatorname{ord}_{w} \mathcal{Z}\right) \delta_{w}$ on $\mathrm{P}^{1}\left(\mathbb{C}_{v}\right)$, still denoted by
$\mathcal{Z}$. Then the diagonal

$$
(\mathcal{Z} \times \mathcal{Z})\left(\operatorname{diag}_{\mathbb{P} 1}(\bar{k})\right)=\sum_{w \in \operatorname{supp}}\left(\operatorname{ord}_{w} \mathcal{Z}\right)^{2}
$$

of $\mathcal{Z}$ is independent of $v \in M_{k}$. For a sequence $\left(\mathcal{Z}_{n}\right)$ of $k$-effective divisors on $\mathbb{P}^{1}(\bar{k})$ satisfying $\lim _{n \rightarrow \infty} \operatorname{deg} \mathcal{Z}_{n}=\infty$, we say $\left(\mathcal{Z}_{n}\right)$ has small g-heights with respect to an adelic normalized weight $g$ if $\lim _{\sup _{n \rightarrow \infty}} h_{g}\left(\mathcal{Z}_{n}\right) \leq 0$, and we say $\left(\mathcal{Z}_{n}\right)$ has small diagonals if $\lim _{n \rightarrow \infty}\left(\left(\mathcal{Z}_{n} \times \mathcal{Z}_{n}\right)\left(\operatorname{diag}_{\mathbb{P}^{1}(\bar{k})}\right)\right) /\left(\operatorname{deg} \mathcal{Z}_{n}\right)^{2}=0$.

1.2. Quantitative adelic equidistribution of effective divisors. The following is one of our main results; for the Galois conjugacy class of an algebraic number, this was due to Favre and Rivera-Letelier [2006, Théorème 7]. For the definitions of the $C^{1}$ regularity of a continuous test function $\phi$ on $\mathrm{P}^{1}\left(\mathbb{C}_{v}\right)$, the Lipschitz constant $\operatorname{Lip}(\phi)_{v}$ on $\left(\mathrm{P}^{1}\left(\mathbb{C}_{v}\right), \mathrm{d}_{v}\right)$, and the Dirichlet norm $\langle\phi, \phi\rangle_{v}$ of $\phi$ for each $v \in M_{k}$, see Section 7.

Theorem 1. Let $k$ be a product formula field and $k_{s}$ the separable closure of $k$ in $\bar{k}$. Let $g=\left\{g_{v}: v \in M_{k}\right\}$ be a placewise Hölder continuous adelic normalized weight. Then for every $v \in M_{k}$, there is $C>0$ such that for every $k$-effective divisor $\mathcal{Z}$ on $\mathbb{P}^{1}\left(k_{s}\right)$ and every test function $\phi \in C^{1}\left(\mathrm{P}^{1}\left(\mathbb{C}_{v}\right)\right)$,

$$
\begin{aligned}
& \left|\int_{\mathrm{P}^{1}\left(\mathbb{C}_{v}\right)} \phi \mathrm{d}\left(\frac{\mathcal{Z}}{\operatorname{deg} \mathcal{Z}}-\mu_{v}^{g}\right)\right| \leq \\
& C \cdot \max \left\{\operatorname{Lip}(\phi)_{v},\langle\phi, \phi\rangle_{v}^{1 / 2}\right\} \sqrt{\max \left\{h_{g}(\mathcal{Z}),(\log \operatorname{deg} \mathcal{Z}) \frac{(\mathcal{Z} \times \mathcal{Z})\left(\operatorname{diag}_{\mathbb{P}^{1}\left(k_{s}\right)}\right)}{(\operatorname{deg} \mathcal{Z})^{2}}\right\} .}
\end{aligned}
$$

In Theorem 1 , if $v \in M_{k}$ is an infinite place, or equivalently, $\mathbb{C}_{v} \cong \mathbb{C}$, then the estimate (1-2) gives a quantitative estimate of the Kantorovich-Wasserstein metric

$$
W\left(\frac{\mathcal{Z}}{\operatorname{deg} \mathcal{Z}}, \mu_{v}^{g}\right)=\sup _{\phi}\left|\int_{\mathbb{P}^{1}(\mathbb{C})} \phi \mathrm{d}\left(\frac{\mathcal{Z}}{\operatorname{deg} \mathcal{Z}}-\mu_{v}^{g}\right)\right|
$$

between the probability Radon measures $\mathcal{Z} / \operatorname{deg} \mathcal{Z}$ and $\mu_{v}^{g}$ on $\mathrm{P}^{1}\left(\mathbb{C}_{v}\right) \cong \mathbb{P}^{1}(\mathbb{C})$, where $\phi$ ranges over all Lipschitz continuous functions on $\mathbb{P}^{1}(\mathbb{C})$ whose Lipschitz 
constants equal 1 with respect to the normalized chordal metric $[z, w]$ on $\mathbb{P}^{1}(\mathbb{C})$ (see Remark 4.2). For the details of the metric $W$ including its role in the optimal transportation problems, see, e.g., [Villani 2009].

The next theorem is a qualitative version of Theorem 1. For a sequence of Galois conjugacy classes of algebraic numbers, this was due to Baker and Rumely [2006, Theorem 2.3], Chambert-Loir [2006, Théorème 4.2], and Favre and RiveraLetelier [2006, Théorème 2]; see also [Szpiro, Ullmo, and Zhang 1997; Bilu 1997; Rumely 1999; Chambert-Loir 2000; Autissier 2001; Baker and Hsia 2005; Baker and Rumely 2006; Chambert-Loir 2006; Favre and Rivera-Letelier 2006], and, most recently, [Yuan 2008] on big line bundles over arithmetic varieties.

Theorem 2 (asymptotically Fekete configuration of effective divisors). Let $k$ be a product formula field and $k_{s}$ its separable closure in $\bar{k}$. Let $g=\left\{g_{v}: v \in M_{k}\right\}$ be an adelic normalized weight. If a sequence $\left(\mathcal{Z}_{n}\right)$ of $k$-effective divisors on $\mathbb{P}^{1}\left(k_{s}\right)$ satisfying $\lim _{n \rightarrow \infty} \operatorname{deg} \mathcal{Z}_{n}=\infty$ has both small diagonals and small g-heights, then for every $v \in M_{k},\left(\mathcal{Z}_{n}\right)$ is an asymptotically $g_{v}$-Fekete configuration on $\mathrm{P}^{1}\left(\mathbb{C}_{v}\right)$. In particular, $\lim _{n \rightarrow \infty} \mathcal{Z}_{n} / \operatorname{deg} \mathcal{Z}_{n}=\mu_{v}^{g}$ weakly on $\mathrm{P}^{1}\left(\mathbb{C}_{v}\right)$.

In Theorem 2 , the assertion that $\left(\mathcal{Z}_{n}\right)$ is an asymptotically $g_{v}$-Fekete configuration on $\mathrm{P}^{1}\left(\mathbb{C}_{v}\right)$ (see (2-7) for the definition), which is also called a $g_{v}$-pseudoequidistribution on $\mathrm{P}^{1}\left(\mathbb{C}_{v}\right)$, is stronger than the final equidistribution assertion. For a relationship between the Kantorovich-Wasserstein metric $W$ and (asymptotically) Fekete configurations on complex manifolds, see [Lev and Ortega-Cerdà 2012, §7]. For a recent result on the capacity and the transfinite diameter on complex manifolds, see [Berman and Boucksom 2010] (on $\mathbb{C}^{n}$, we also refer to the survey [Levenberg 2010]); for the convergence of (asymptotically) Fekete points on complex manifolds, see [Berman, Boucksom, and Nyström 2011].

1.3. Quantitative equidistribution in adelic dynamics. For rational functions $f, a$ over a field $k$ and for $n \in \mathbb{N}$, the divisor $\left[f^{n}=a\right]$ defined by the roots of the equation $f^{n}=a$ in $\mathbb{P}^{1}(\bar{k})$ is a $k$-effective divisor on $\mathbb{P}^{1}(\bar{k})$ if $f^{n} \not \equiv a$.

Let $k$ be a product formula field. For a rational function $f \in k(z)$ of degree $d>1$, let $\hat{g}_{f}:=\left\{g_{f, v}: v \in M_{k}\right\}$ be the adelic dynamical Green function in the sense that for every $v \in M_{k}, g_{f, v}$ is the dynamical Green function of $f$ on $\mathrm{P}^{1}\left(\mathbb{C}_{v}\right)$, so that $\mu_{f, v}:=\mu^{g_{f, v}}$ is the $f$-equilibrium (or canonical) measure on $\mathrm{P}^{1}\left(\mathbb{C}_{v}\right)$ (see Section 9 for details). The family $\hat{g}_{f}$ is in fact an adelic normalized weight, and the $\hat{g}_{f}$-height function $h_{\hat{g}_{f}}$ coincides with the Call-Silverman $f$-dynamical (or canonical) height function. For every rational function $a \in k(z)$, the sequence ([ $\left.f^{n}=a\right]$ ) has strictly small $\hat{g}_{f}$-heights in that $\lim \sup _{n \rightarrow \infty}\left(d^{n}+\operatorname{deg} a\right) \cdot h_{\hat{g}_{f}}\left(\left[f^{n}=a\right]\right)<\infty$ (Lemma 9.2). Hence the following are consequences of Theorems 1 and 2, respectively.

Theorem 3. Let $k$ be a product formula field and $k_{s}$ its separable closure in $\bar{k}$. Let $f \in k(z)$ be a rational function of degree $d>1$ and $a \in k(z)$ a rational function. 
Then for every $v \in M_{k}$, there exists a constant $C>0$ such that for every test function $\phi \in C^{1}\left(\mathrm{P}^{1}\left(\mathbb{C}_{v}\right)\right)$ and every $n \in \mathbb{N}$,

$$
\begin{aligned}
& \left|\int_{\mathrm{P}^{1}\left(\mathbb{C}_{v}\right)} \phi \mathrm{d}\left(\frac{\left[f^{n}=a\right]}{d^{n}+\operatorname{deg} a}-\mu_{f, v}\right)\right| \\
& \quad \leq C \cdot \max \left\{\operatorname{Lip}(\phi)_{v},\langle\phi, \phi\rangle_{v}^{1 / 2}\right\} \sqrt{\frac{n \cdot\left(\left[f^{n}=a\right] \times\left[f^{n}=a\right]\right)\left(\operatorname{diag}_{\mathbb{P}^{1}\left(k_{s}\right)}\right)}{\left(d^{n}+\operatorname{deg} a\right)^{2}}}
\end{aligned}
$$

if $f^{n} \not \equiv a$ and the divisor $\left[f^{n}=a\right]$ on $\mathbb{P}^{1}(\bar{k})$ is on $\mathbb{P}^{1}\left(k_{s}\right)$.

Theorem 4. Let $k$ be a product formula field and $k_{s}$ its separable closure in $\vec{k}$. Let $f \in k(z)$ be a rational function of degree $d>1$ and $a \in k(z)$ a rational function. If the sequence $\left(\left[f^{n}=a\right]\right)$ has small diagonals and the divisor $\left[f^{n}=a\right]$ is on $\mathbb{P}^{1}\left(k_{s}\right)$ for every sufficiently large $n \in \mathbb{N}$, then for every $v \in M_{k}$, $\left(\left[f^{n}=a\right]\right)$ is an asymptotically $g_{f, v}$-Fekete configuration on $\mathrm{P}^{1}\left(\mathbb{C}_{v}\right)$. In particular,

$$
\lim _{n \rightarrow \infty} \frac{\left[f^{n}=a\right]}{d^{n}+\operatorname{deg} a}=\mu_{f, v}
$$

weakly on $\mathrm{P}^{1}\left(\mathbb{C}_{v}\right)$.

The final equidistribution assertion in Theorem 4 has been established in [Brolin 1965; Ljubich 1983; Freire, Lopes, and Mañé 1983] in complex dynamics, and in [Favre and Rivera-Letelier 2010] in (not necessarily adelic) nonarchimedean dynamics (of characteristic 0 when $\operatorname{deg} a>0$ ). For every constant $a \in \mathbb{P}^{1}(k)$, the estimate (1-3) in Theorem 3 has been obtained in [Okuyama 2013b, Theorems 4 and 5] in complex and (not necessarily adelic) nonarchimedean dynamics of characteristic 0 . In complex dynamics, for every $f \in \mathbb{C}(z)$ of degree $d>1$, every constant $a \in \mathbb{P}^{1}(\mathbb{C})$, and every $\phi \in C^{2}\left(\mathbb{P}^{1}(\mathbb{C})\right)$, a finer estimate than (1-3) has been obtained in [Drasin and Okuyama 2007, Theorem 2 and (4.2)].

1.4. Application to a motivating question. Let $K$ be an algebraically closed field that is complete with respect to a nontrivial absolute value $|\cdot|$, and $[z, w]$ be the normalized chordal metric on $\mathbb{P}^{1}=\mathbb{P}^{1}(K)$ (see (2-1)). A subset $D$ in $\mathbb{P}^{1}$ is called a chordal disk (in $\mathbb{P}^{1}$ ) if $D=\left\{z \in \mathbb{P}^{1}:[z, w] \leq r\right\}$ for some $w \in \mathbb{P}^{1}$ and some radius $r \geq 0$. Even in the specific case $a=\mathrm{Id}$ (see, e.g., [Cremer 1928; Siegel 1942; Brjuno 1971; 1972; Herman and Yoccoz 1983; Yoccoz 1988; 1995; Pérez-Marco 1993; 2001]), which is one of the most interesting cases and is related to the difficulty of small denominators in nonarchimedean and complex dynamics, the following question has not been completely understood.

Question. How uniformly close on a chordal disk $D$ of radius $>0$ can the sequence $\left(f^{n}\right)$ of the iterations of a rational function $f \in K(z)$ of degree $>1$ be to a rational function $a \in K(z)$ of degree $>0$ ? 
For a study of this question on the projective space $\mathbb{P}^{N}(K)$, see [Okuyama 2010]. The following estimate of the local proximity sequence $\left(\sup _{D}\left[f^{n}, a\right]_{v}\right.$ ) is an application of Theorem 3 to this question in the setting of adelic dynamics.

Theorem 5. Let $k$ be a product formula field of characteristic 0 . Let $f \in k(z)$ be a rational function of degree $>1$ and $a \in k(z)$ a rational function of degree $>0$. Then for every $v \in M_{k}$ and every chordal disk $D$ in $\mathbb{P}^{1}\left(\mathbb{C}_{v}\right)$ of radius $>0$, as $n \rightarrow \infty$,

$$
\left.\log \sup _{D}\left[f^{n}, a\right]_{v}=O\left(\sqrt{n \cdot\left(\left[f^{n}=a\right] \times\left[f^{n}=a\right]\right)\left(\operatorname{diag}_{\mathbb{P} 1}(\bar{k})\right.}\right)\right) .
$$

Here, the implicit constant in $O(\cdot)$ possibly depends on $f$ and $a$.

In the case that $a=\mathrm{Id}$, we will see that $\left(\left[f^{n}=\mathrm{Id}\right] \times\left[f^{n}=\mathrm{Id}\right]\right)\left(\operatorname{diag}_{\mathbb{P}^{1}(\bar{k})}\right)=O\left(d^{n}\right)$ as $n \rightarrow \infty$ in Section 10. Hence Theorem 5 concludes the following.

Theorem 6. Let $k$ be a product formula field of characteristic 0 . Let $f \in k(z)$ be a rational function of degree $d>1$. Then for every $v \in M_{k}$ and every chordal disk $D$ in $\mathbb{P}^{1}\left(\mathbb{C}_{v}\right)$ of radius $>0$,

$$
\log \sup _{D}\left[f^{n}, \mathrm{Id}\right]_{v}=O\left(\sqrt{n d^{n}}\right) \quad \text { as } n \rightarrow \infty .
$$

1.5. The unit $D^{*}(p)$. The next result generalizes the obvious fact that the discriminant of a polynomial in one variable over a field $k$ is in $k$. The unit $D^{*}(p)$ plays an important role in the nonseparable case and might have been studied before, but we could find no relevant literature.

Theorem 7. Let $k$ be a field and $k_{s}$ the separable closure of $k$ in an algebraic closure $\bar{k}$ of $k$. For every $p(z) \in k[z]$ of degree $>0$, let $\left\{z_{1}, \ldots, z_{m}\right\}$ be the set of all distinct zeros of $p(z)$ in $\bar{k}$ so that $p(z)=a \cdot \prod_{j=1}^{m}\left(z-z_{j}\right)^{d_{j}}$ in $\bar{k}[z]$ for some $a \in k \backslash\{0\}$ and some sequence $\left(d_{j}\right)_{j=1}^{m}$ in $\mathbb{N}$. If $\left\{z_{1}, \ldots, z_{m}\right\} \subset k_{s}$, then

$$
D^{*}(p):=\prod_{j=1}^{m} \prod_{i: i \neq j}\left(z_{j}-z_{i}\right)^{d_{i} d_{j}} \in k \backslash\{0\},
$$

where, a priori, this $D^{*}(p)$ is always in $\bar{k} \backslash\{0\}$.

1.6. Organization of this article. In Section 2, we recall background from potential theory and arithmetic on the Berkovich projective line. In Section 3, we extend Favre and Rivera-Letelier's regularization $[\cdot]_{\epsilon}$ of discrete Radon measures and establish required estimates on them, and in Section 4 we see the negativity of regularized Fekete sums and a Cauchy-Schwarz inequality. In Sections 5 and 6 , we compute the $g$-Fekete sums $(\mathcal{Z}, \mathcal{Z})_{g}$ and estimate the regularized $g$-Fekete sums $\left(\mathcal{Z}_{\epsilon}, \mathcal{Z}_{\epsilon}\right)_{g}$ with respect to a $k$-effective divisor $\mathcal{Z}$ on $\mathbb{P}^{1}(\bar{k})$. In Section 7 , we prove Theorems 1 and 2; the arguments are more or less adaptions of those in the 
proofs of [Favre and Rivera-Letelier 2006, Théorème 7] and [Baker and Rumely 2010, Theorem 10.24], respectively. In Section 8, we review background from nonarchimedean and complex dynamics. Finally, we prove Theorems 3 and 4 in Section 9, Theorems 5 and 6 in Section 10, and Theorem 7 in Section 11.

\section{Background from potential theory and arithmetic}

Notation 2.1. For a field $k$, the origin of $k^{2}$ is also denoted by $0=0_{k}$, and we write $\pi=\pi_{k}: k^{2} \backslash\{0\} \rightarrow \mathbb{P}^{1}=\mathbb{P}^{1}(k)$ for the canonical projection, so that $\pi(0,1)=\infty$ and $\pi\left(p_{0}, p_{1}\right)=p_{1} / p_{0}$ if $p_{0} \neq 0$. Set the wedge product $\left(z_{0}, z_{1}\right) \wedge\left(w_{0}, w_{1}\right):=$ $z_{0} w_{1}-z_{1} w_{0}$ on $k^{2}$.

Let $K$ be an algebraically closed field that is complete with respect to a nontrivial absolute value $|\cdot|$, which is said to be nonarchimedean if the strong triangle inequality $|z+w| \leq \max \{|z|,|w|\}$ holds, and archimedean otherwise. On $K^{2}$, let $\left\|\left(p_{0}, p_{1}\right)\right\|$ be either the maximal norm $\max \left\{\left|p_{0}\right|,\left|p_{1}\right|\right\}$ (for nonarchimedean $K$ ) or the euclidean norm $\sqrt{\left|p_{0}\right|^{2}+\left|p_{1}\right|^{2}}$ (for archimedean $K$ ). The normalized chordal metric $[z, w]$ on $\mathbb{P}^{1}=\mathbb{P}^{1}(K)$ is the function

$$
(z, w) \mapsto[z, w]=|p \wedge q| /(\|p\| \cdot\|q\|) \leq 1
$$

on $\mathbb{P}^{1} \times \mathbb{P}^{1}$, where $p \in \pi^{-1}(z), q \in \pi^{-1}(w)$. The metric topology on $\mathbb{P}^{1}$ with respect to $[z, w]$ agrees with the relative topology on $\mathbb{P}^{1}$ from the Berkovich projective line $\mathrm{P}^{1}=\mathrm{P}^{1}(K)$, which is a compact augmentation of $\mathbb{P}^{1}$ containing $\mathbb{P}^{1}$ as a dense subset, and is isomorphic to $\mathbb{P}^{1}$ if and only if $K$ is archimedean (see Section 3.2 for more details when $K$ is nonarchimedean). Letting $\delta_{\mathcal{S}}$ be the Dirac measure on $\mathrm{P}^{1}$ at a point $\mathcal{S} \in \mathrm{P}^{1}$, set

$$
\Omega_{\text {can }}:= \begin{cases}\delta_{\mathcal{S}_{\text {can }}} & \text { for nonarchimedean } K, \\ \omega & \text { for } \operatorname{archimedean} K\end{cases}
$$

where $\mathcal{S}_{\text {can }}$ is the canonical (or Gauss) point in $\mathrm{P}^{1}$ for nonarchimedean $K$ (see Section 3.2 for the definition), and $\omega$ is the Fubini-Study area element on $\mathbb{P}^{1}$ normalized as $\omega\left(\mathbb{P}^{1}\right)=1$ for archimedean $K$. For nonarchimedean $K$, the generalized Hsia kernel $\left[\mathcal{S}, \mathcal{S}^{\prime}\right]_{\text {can }}$ on $\mathrm{P}^{1}$ with respect to $\mathcal{S}_{\text {can }}$ is the unique (jointly) upper semicontinuous and separately continuous extension of the normalized chordal metric $[z, w]$ on $\mathbb{P}^{1}\left(\times \mathbb{P}^{1}\right)$ to $\mathrm{P}^{1} \times \mathrm{P}^{1}$ (see (3-4) for a more concrete description). By convention, for archimedean $K$, the kernel function $\left[\mathcal{S}, \mathcal{S}^{\prime}\right]_{\text {can }}$ is defined by $[z, w]$ itself. Let $\Delta=\Delta_{\mathrm{P}^{1}}$ be the distributional Laplacian on $\mathrm{P}^{1}$ normalized so that for each $\mathcal{S}^{\prime} \in \mathrm{P}^{1}$,

$$
\Delta \log \left[\cdot, \mathcal{S}^{\prime}\right]_{\mathrm{can}}=\delta_{\mathcal{S}^{\prime}}-\Omega_{\mathrm{can}} \quad \text { on } \mathrm{P}^{1} .
$$


For the construction of the Laplacian $\Delta$ in the nonarchimedean case, see [Baker and Rumely 2010, §5; Favre and Jonsson 2004, §7.7; Thuillier 2005, §3] and also [Jonsson 2015, §2.5]. In [Baker and Rumely 2010], the opposite sign convention for $\Delta$ is adopted.

2.1. Potential theory on $\mathbf{P}^{1}$ with external fields. For the foundation of the potential theory on the (Berkovich) projective line, see [Baker and Rumely 2010; Favre and Rivera-Letelier 2010; Thuillier 2005], and also [Jonsson 2015; Tsuji 1959, III §11] ([Thuillier 2005] is on more general curves than lines and [Tsuji 1959, III §11] is on $\mathbb{P}^{1}(\mathbb{C})$ ). We also refer to [Saff and Totik 1997] for the generalities of weighted potential theory, i.e., logarithmic potential theory with external fields.

A continuous weight $g$ on $\mathrm{P}^{1}$ is a continuous function on $\mathrm{P}^{1}$ such that

$$
\mu^{g}:=\Delta g+\Omega_{\text {can }}
$$

is a probability Radon measure on $\mathrm{P}^{1}$. For a continuous weight $g$ on $\mathrm{P}^{1}$, the $g$ potential kernel on $\mathrm{P}^{1}$ (or the negative of an Arakelov Green kernel function on $\mathrm{P}^{1}$ relative to $\mu^{g}$ [Baker and Rumely 2010, §8.10]) is the function

$$
\Phi_{g}\left(\mathcal{S}, \mathcal{S}^{\prime}\right):=\log \left[\mathcal{S}, \mathcal{S}^{\prime}\right]_{\mathrm{can}}-g(\mathcal{S})-g\left(\mathcal{S}^{\prime}\right) \quad \text { on } \mathrm{P}^{1} \times \mathrm{P}^{1},
$$

and the $g$-potential of a Radon measure $v$ on $\mathrm{P}^{1}$ is the function

$$
U_{g, v}(\cdot):=\int_{\mathrm{P}^{1}} \Phi_{g}\left(\cdot, \mathcal{S}^{\prime}\right) \mathrm{d} \nu\left(\mathcal{S}^{\prime}\right) \quad \text { on } \mathrm{P}^{1} .
$$

By Fubini's theorem, $\Delta U_{g, v}=v-v\left(\mathrm{P}^{1}\right) \mu^{g}$ on $\mathrm{P}^{1}$. The g-equilibrium energy $V_{g} \in(-\infty,+\infty)$ of $\mathrm{P}^{1}$ is the supremum of the $g$-energy functional

$$
v \mapsto \int_{\mathrm{P}^{1} \times \mathrm{P}^{1}} \Phi_{g} \mathrm{~d}(v \times v)=\int_{\mathrm{P}^{1}} U_{g, v} \mathrm{~d} v
$$

on the space of all probability Radon measures $v$ on $\mathrm{P}^{1}$; indeed, $V_{g}>-\infty$ since $V_{g} \geq \int_{\mathrm{P}^{1} \times \mathrm{P}^{1}} \Phi_{g} \mathrm{~d}\left(\Omega_{\text {can }} \times \Omega_{\text {can }}\right)>-\infty$. A probability Radon measure $\mu$ on $\mathrm{P}^{1}$ at which the $g$-energy functional (2-6) attains the supremum $V_{g}$ is called a $g$-equilibrium mass distribution on $\mathrm{P}^{1}$; in fact the unique $g$-equilibrium mass distribution on $\mathrm{P}^{1}$ is $\mu^{g}$, and moreover, $U_{g, \mu^{g}} \equiv V_{g}$ on $\mathrm{P}^{1}$ (for nonarchimedean $K$, see [Baker and Rumely 2010, Theorem 8.67, Proposition 8.70]). For a discussion on such a Gauss variational problem, see [Saff and Totik 1997, Chapter 1].

A normalized weight $g$ on $\mathrm{P}^{1}$ is a continuous weight on $\mathrm{P}^{1}$ satisfying $V_{g}=0$; for every continuous weight $g$ on $\mathrm{P}^{1}, \bar{g}:=g+V_{g} / 2$ is the unique normalized weight on $\mathrm{P}^{1}$ such that $\mu^{\bar{g}}=\mu^{g}$.

For a continuous weight $g$ on $\mathrm{P}^{1}$ and a Radon measure $v$ on $\mathrm{P}^{1}$, the $g$-Fekete 
sum with respect to $v$ is

$$
(v, v)_{g}:=\int_{\mathrm{P}^{1} \times \mathrm{P}^{1} \backslash \operatorname{diag}_{\mathbb{P}^{1}(K)}} \Phi_{g} \mathrm{~d}(v \times v),
$$

which generalizes the classical Fekete sum associated with a finite subset in $\mathbb{C}$ (see [Fekete 1930a; 1930b; 1933]). If supp $v$ is a discrete (so finite) subset in $\mathrm{P}^{1}$, i.e., if $v$ is a discrete measure on $\mathrm{P}^{1}$, then $(v, v)_{g}$ is always finite (even if $\left.\operatorname{supp} v \subset \mathbb{P}^{1}\right)$.

For a continuous weight $g$ on $\mathrm{P}^{1}$, a sequence $\left(v_{n}\right)$ of positive and discrete Radon measures on $\mathrm{P}^{1}$ satisfying $\lim _{n \rightarrow \infty} v_{n}\left(\mathrm{P}^{1}\right)=\infty$ is called an asymptotically g-Fekete configuration on $\mathrm{P}^{1}$ if the sequence $\left(v_{n}\right)$ not only has small diagonals in that $\left(v_{n} \times v_{n}\right)\left(\operatorname{diag}_{\mathbb{P}^{1}(K)}\right)=o\left(v_{n}\left(\mathrm{P}^{1}\right)^{2}\right)$ as $n \rightarrow \infty$ but also satisfies $\lim _{n \rightarrow \infty}\left(v_{n}, v_{n}\right)_{g} /\left(v_{n}\left(\mathrm{P}^{1}\right)\right)^{2}=V_{g}$; under the former small diagonals condition, the latter one is equivalent to the weaker

$$
\liminf _{n \rightarrow \infty} \frac{\left(v_{n}, v_{n}\right)_{g}}{\left(v_{n}\left(\mathrm{P}^{1}\right)\right)^{2}} \geq V_{g}
$$

since we always have

$$
\limsup _{n \rightarrow \infty} \frac{\left(v_{n}, v_{n}\right)_{g}}{\left(v_{n}\left(\mathrm{P}^{1}\right)\right)^{2}} \leq V_{g}
$$

(see, e.g., [Baker and Rumely 2010, Lemma 7.54]). By a classical argument (see [Saff and Totik 1997, Theorem 1.3 in Chapter III]), if $\left(v_{n}\right)$ is an asymptotically $g$-Fekete configuration on $\mathrm{P}^{1}$, then $\lim _{n \rightarrow \infty} v_{n} / v_{n}\left(\mathrm{P}^{1}\right)=\mu^{g}$ weakly on $\mathrm{P}^{1}$.

\subsection{Local arithmetic on $\mathbf{P}^{1}$. Let $k$ be a field.}

Definition 2.2. A field extension $K / k$ is an algebraic and metric augmentation of $k$ if $K$ is algebraically closed and (topologically) complete with respect to a nontrivial absolute value $|\cdot|$ (e.g., $\mathbb{C}_{v}$ is an algebraic and metric augmentation of a product formula field $k$ for every $v \in M_{k}$ ).

For every $P \in \bigcup_{d \in \mathbb{N}} k\left[p_{0}, p_{1}\right]_{d}$, there is a sequence $\left(q_{j}^{P}\right)_{j=1}^{\operatorname{deg} P}$ in $\bar{k}^{2} \backslash\{0\}$ giving a factorization

$$
P\left(p_{0}, p_{1}\right)=\prod_{j=1}^{\operatorname{deg} P}\left(\left(p_{0}, p_{1}\right) \wedge q_{j}^{P}\right)
$$

of $P$ in $\bar{k}\left[p_{0}, p_{1}\right]$. Set $z_{j}^{P}:=\pi\left(q_{j}^{P}\right) \in \mathbb{P}^{1}(\bar{k})$ for each $j \in\{1,2, \ldots, \operatorname{deg} P\}$. Although the sequence $\left(q_{j}^{P}\right)_{j=1}^{\operatorname{deg} P}$ is not unique, the sequence $\left(z_{j}^{P}\right)_{j=1}^{\operatorname{deg} P}$ in $\mathbb{P}^{1}(\bar{k})$ is independent of the choice of $\left(q_{j}^{P}\right)_{j=1}^{\operatorname{deg} P}$ up to permutations. Let in addition $K$ be an algebraic and metric completion of $k$. Then the $\operatorname{sum} M^{\#}(P):=\sum_{j=1}^{\operatorname{deg} P} \log \left\|q_{j}^{P}\right\|$ is also independent of the choice of $\left(q_{j}^{P}\right)_{j=1}^{\operatorname{deg} P}$, and for every continuous weight $g$ on $\mathrm{P}^{1}=\mathrm{P}^{1}(K)$, the logarithmic $g$-Mahler measure of $P$ is 


$$
M_{g}(P):=\sum_{j=1}^{\operatorname{deg} P} g\left(z_{j}^{P}\right)+M^{\#}(P) .
$$

The function $S_{P}:=|P(\cdot /\|\cdot\|)|$ on $K^{2} \backslash\{0\}$ descends to $\mathbb{P}^{1}(K)$ and in turn extends continuously to $\mathrm{P}^{1}$ so that $\log S_{P}=\sum_{j=1}^{\operatorname{deg} P} \log \left[\cdot, z_{j}^{P}\right]_{\text {can }}+M^{\#}(P)$ on $\mathrm{P}^{1}$, which can be rewritten as $\log S_{P}-(\operatorname{deg} P) g=\sum_{j=1}^{\operatorname{deg} P} \Phi_{g}\left(\cdot, z_{j}^{P}\right)+M_{g}(P)$ on $\mathrm{P}^{1}$. Integrating both sides against $\mathrm{d} \mu^{g}$ over $\mathrm{P}^{1}$, by $U_{g, \mu^{g}} \equiv V_{g}$ on $\mathrm{P}^{1}$, we have the Jensen-type formula

$$
M_{g}(P)=\int_{\mathrm{P}^{1}}\left(\log S_{P}-(\operatorname{deg} P) g\right) \mathrm{d} \mu^{g}-(\operatorname{deg} P) V_{g} .
$$

2.3. A lemma on global arithmetic. Let $k$ be a product formula field. The proof of the next result is not based on a field extension of $k$.

Lemma 2.3. For every $P \in \bigcup_{d \in \mathbb{N}} k\left[p_{0}, p_{1}\right]_{d}$, we have $\sum_{v \in M_{k}} N_{v} \cdot M^{\#}(P)_{v} \in \mathbb{R}_{\geq 0}$.

Proof. Let $\left(q_{j}^{P}\right)_{j=1}^{\operatorname{deg} P}$ be a sequence in $\bar{k}^{2} \backslash\{0\}$ giving a factorization (2-9) of $P$, and let $L(P(1, \cdot)) \in k \backslash\{0\}$ be the coefficient of the maximal degree term of $P(1, z) \in k[z]$. Setting $q_{j}^{P}=\left(\left(q_{j}^{P}\right)_{0},\left(q_{j}^{P}\right)_{1}\right)$, for each $j \in\{1,2, \ldots, \operatorname{deg} P\}$, we have

$$
L(P(1, \cdot))=(-1)^{\operatorname{deg} P-\operatorname{deg}_{\infty} P}\left(\prod_{j: \pi\left(q_{j}^{P}\right)=\infty}\left(q_{j}^{P}\right)_{1}\right)\left(\prod_{j: \pi\left(q_{j}^{P}\right) \neq \infty}\left(q_{j}^{P}\right)_{0}\right)
$$

since for each $j \in\{1,2, \ldots, \operatorname{deg} P\}$,

$$
q_{j}^{P}= \begin{cases}\left(q_{j}^{P}\right)_{0} \cdot\left(1, \pi\left(q_{j}^{P}\right)\right) & \text { if } \pi\left(q_{j}^{P}\right) \neq \infty, \\ \left(q_{j}^{P}\right)_{1} \cdot(0,1) & \text { if } \pi\left(q_{j}^{P}\right)=\infty .\end{cases}
$$

Thus we have $\sum_{v \in M_{k}} N_{v} \cdot M^{\#}(P)_{v} \geq \sum_{v \in M_{k}} N_{v} \log |L(P(1, \cdot))|_{v}=0$, where the final equality is by $(\mathrm{PF})$.

For each $i, j \in \mathbb{N} \cup\{0\}$ satisfying $i+j=\operatorname{deg} P$, if the coefficient $a_{i, j} \in k$ of the expansion $P\left(p_{0}, p_{1}\right)=\sum_{i+j=\operatorname{deg} P} a_{i, j} p_{0}^{i} p_{1}^{j}$ in $k\left[p_{0}, p_{1}\right]_{\operatorname{deg} P}$ does not vanish, then by (PF), there is a finite subset $E_{i, j}$ in $M_{k}$ such that $\left|a_{i, j}\right|_{v}=1$ for every $v \in M_{k} \backslash E_{i, j}$. Set $E_{P}:=\{$ infinite places of $k\} \cup \bigcup_{i, j \in \mathbb{N} \cup\{0\}: a_{i, j} \neq 0} E_{i, j}$. For every $v \in M_{k} \backslash E_{P}$, by the strong triangle inequality, $\left|P\left(p_{0}, p_{1}\right)\right|_{v}$ is bounded above by

$$
\max \left\{\max \left\{\left|p_{0}\right|_{v},\left|p_{1}\right|_{v}\right\}^{i+j}: i, j \in \mathbb{N} \cup\{0\}, i+j=\operatorname{deg} P\right\}=\left\|\left(p_{0}, p_{1}\right)\right\|_{v}^{\operatorname{deg} P}
$$

on $\mathbb{C}_{v}^{2}$, so that $\log S_{P, v} \leq 0$ on $\mathbb{P}^{1}\left(\mathbb{C}_{v}\right)$ and in turn on $\mathrm{P}^{1}\left(\mathbb{C}_{v}\right)$. Set $g^{0}:=\left\{g_{v}^{0}: v \in M_{k}\right\}$ with $g_{v}^{0} \equiv 0$ on $\mathrm{P}^{1}\left(\mathbb{C}_{v}\right)$ for every $v \in M_{k}$; then $g^{0}$ is an adelic continuous weight. For every finite $v \in M_{k}$, we have $\mu_{v}^{g^{0}}=\delta_{\mathcal{S}_{\text {can }, v}}$ on $\mathrm{P}^{1}\left(\mathbb{C}_{v}\right)$ and moreover $V_{g_{v}^{0}}=$ $\log \left[\mathcal{S}_{\text {can }, v}, \mathcal{S}_{\text {can }, v}\right]_{\text {can }, v}=0$, so that by the Jensen-type formula (2-11), we have $M^{\#}(P)_{v}=M_{g_{v}^{0}}(P)=\log S_{P, v}\left(\mathcal{S}_{\text {can }, v}\right)$. Hence, $M^{\#}(P)_{v} \leq 0$ for every $v \in M_{k} \backslash E_{P}$, and we conclude that $\sum_{v \in M_{k}} N_{v} \cdot M^{\#}(P)_{v}<\infty$ since $\# E_{P}<\infty$. 


\section{Regularization of discrete Radon measures whose supports are in $\mathbb{P}^{1}$}

Let $K$ be an algebraically closed field complete with respect to a nontrivial absolute value $|\cdot|$.

3.1. The small model metric $\mathrm{d}$ and the Hsia kernel $\left|\mathcal{S}-\mathcal{S}^{\prime}\right|_{\infty}$. The kernel function $\left[\mathcal{S}, \mathcal{S}^{\prime}\right]_{\text {can }}$ is not necessarily a metric on $\mathrm{P}^{1}=\mathrm{P}^{1}(K)$; indeed, for every $\mathcal{S} \in \mathrm{P}^{1}$, $[\mathcal{S}, \mathcal{S}]_{\text {can }}$ vanishes if and only if $\mathcal{S} \in \mathbb{P}^{1}=\mathbb{P}^{1}(K)$. The small model metric $\mathrm{d}$ on $\mathrm{P}^{1}$ is the function

$$
\mathrm{d}\left(\mathcal{S}, \mathcal{S}^{\prime}\right):=\left[\mathcal{S}, \mathcal{S}^{\prime}\right]_{\mathrm{can}}-\frac{[\mathcal{S}, \mathcal{S}]_{\mathrm{can}}+\left[\mathcal{S}^{\prime}, \mathcal{S}^{\prime}\right]_{\mathrm{can}}}{2} \quad \text { on } \mathrm{P}^{1} \times \mathrm{P}^{1},
$$

which extends the normalized chordal metric $[z, w]$ on $\mathbb{P}^{1}$ (but this $\mathrm{d}$ does not induce the topology of $\mathrm{P}^{1}$; see [Baker and Rumely 2010, §2.7; Favre and RiveraLetelier 2006, §4.7] for details). On the other hand, the Hsia kernel $\left|\mathcal{S}-\mathcal{S}^{\prime}\right|_{\infty}$ on the Berkovich affine line $\mathrm{A}^{1}=\mathrm{A}^{1}(K)=\mathrm{P}^{1} \backslash\{\infty\}$ is the function

$$
\left|\mathcal{S}-\mathcal{S}^{\prime}\right|_{\infty}:=\left[\mathcal{S}, \mathcal{S}^{\prime}\right]_{\text {can }} \cdot[\mathcal{S}, \infty]_{\text {can }}^{-1} \cdot\left[\mathcal{S}^{\prime}, \infty\right]_{\text {can }}^{-1} \text { on } A^{1} \times A^{1},
$$

although the difference $\mathcal{S}-\mathcal{S}^{\prime}$ itself is not defined unless both $\mathcal{S}, \mathcal{S}^{\prime} \in K$ (for details, see [Baker and Rumely 2010, Chapter 4]). The kernel $\left|\mathcal{S}-\mathcal{S}^{\prime}\right|_{\infty}$ is the unique (jointly) upper semicontinuous and separately continuous extension of the function $|z-w|$ on $K \times K$ to $\mathrm{A}^{1} \times \mathrm{A}^{1}$.

3.2. A short description of $\mathrm{P}^{1}$ for nonarchimedean $K$. Suppose that $K$ is nonarchimedean. A subset $B$ in $K$ is called a ( $K$-closed) disk in $K$ if it has the form $B=\{z \in K:|z-a| \leq r\}$ for some $a \in K$ and some radius $r \geq 0$. By the strong triangle inequality, two disks in $K$ either nest or are disjoint. This alternative extends to any two decreasing infinite sequences of disks in $K$ such that they either infinitely nest or are eventually disjoint, and so induces a cofinal equivalence relation among them.

Example 3.1. Instead of giving a formal definition of the cofinal equivalence class $\mathcal{S}$ of a decreasing infinite sequence $\left(B_{n}\right)$ of disks in $K$, let us be practical: each $z \in K$ is regarded as the cofinal equivalence class of the constant sequence $\left(B_{n}\right)$ of the disks $B_{n} \equiv\{z\}$ in $K$ (of radii $\equiv 0$ ). More generally, for every cofinal equivalence class $\mathcal{S}$ of a decreasing infinite sequence $\left(B_{n}\right)$ of disks in $K$, the intersection $B_{\mathcal{S}}:=\bigcap_{n \in \mathbb{N}} B_{n}$ is independent of the choice of the representatives $\left(B_{n}\right)$ of $\mathcal{S}$, and if $B_{\mathcal{S}} \neq \varnothing$, then $B_{\mathcal{S}}$ is still a disk in $K$ and the $\mathcal{S}$ is represented by the constant sequence $\left(\tilde{B}_{n}\right)$ of the disks $\tilde{B}_{n} \equiv B_{\mathcal{S}}$ in $K$.

As a set, the set of all cofinal equivalence classes $\mathcal{S}$ of decreasing infinite sequences $\left(B_{n}\right)$ of disks in $K$ and in addition $\infty \in \mathbb{P}^{1}$ is nothing but $\mathrm{P}^{1}$ ([Berkovich 1990, p. 17]; see also [Baker and Rumely 2010, §2; Favre and Rivera-Letelier 2006, $\S 3$; Benedetto 2010, §6.1]): for example, the canonical (or Gauss) point $\mathcal{S}_{\text {can }}$ in 
$\mathrm{P}^{1}$ is represented by the ring of $K$-integers $\mathcal{O}_{K}:=\{z \in K:|z| \leq 1\}$, which is a disk in $K$. The above alternative induces a partial ordering $\succeq$ on $\mathrm{P}^{1}$ such that for every $\mathcal{S}, \mathcal{S}^{\prime} \in \mathrm{P}^{1}$ satisfying $B_{\mathcal{S}}, B_{\mathcal{S}^{\prime}} \neq \varnothing$, we have $\mathcal{S} \succeq \mathcal{S}^{\prime}$ if and only if $B_{\mathcal{S}} \supset B_{\mathcal{S}^{\prime}}$ (the description is a little complicated when one of $B_{\mathcal{S}}, B_{\mathcal{S}^{\prime}}$ equals $\varnothing$ ). For every $\mathcal{S}, \mathcal{S}^{\prime} \in \mathrm{P}^{1}$ satisfying $\mathcal{S} \succeq \mathcal{S}^{\prime}$, the segment between $\mathcal{S}$ and $\mathcal{S}^{\prime}$ in $\mathrm{P}^{1}$ is the set of all points $\mathcal{S}^{\prime \prime} \in \mathrm{P}^{1}$ satisfying $\mathcal{S} \succeq \mathcal{S}^{\prime \prime} \succeq \mathcal{S}^{\prime}$, which can be equipped with either the ordering induced by $\succeq$ on $\mathrm{P}^{1}$ or its opposite. All those (oriented) segments make $P^{1}$ a tree in the sense of Jonsson [2015, §2, Definition 2.2]. The (Gelfand) topology of $\mathrm{P}^{1}$ coincides with the (weak) topology of $\mathrm{P}^{1}$ as a tree.

For each $\mathcal{S} \in \mathrm{P}^{1} \backslash\{\infty\}$ represented by $\left(B_{n}\right)$, set

$$
\operatorname{diam} \mathcal{S}:=\lim _{n \rightarrow \infty} \operatorname{diam} B_{n} \quad\left(=\operatorname{diam} B_{\mathcal{S}} \text { if } B_{\mathcal{S}} \neq \varnothing\right),
$$

where $\operatorname{diam} B$ denotes the diameter of a disk $B$ in $K$ with respect to $|\cdot|$; by convention, for $\mathcal{S}=\infty$, we set $B_{\infty}:=K$ and diam $\infty:=+\infty$. The hyperbolic space is $\mathrm{H}^{1}=\mathrm{H}^{1}(K):=\mathrm{P}^{1} \backslash \mathbb{P}^{1}=\left\{\mathcal{S} \in \mathrm{P}^{1}: \operatorname{diam} \mathcal{S} \in(0,+\infty)\right\}$. The big model (or hyperbolic) metric $\rho$ on $\mathrm{H}^{1}$ is a path metric on $\mathrm{H}^{1}$ (but does not induce the relative topology of $\mathrm{H}^{1}$ induced by $\mathrm{P}^{1}$ ) so that for every $\mathcal{S}, \mathcal{S}^{\prime} \in \mathrm{H}^{1}$ satisfying $\mathcal{S} \succeq \mathcal{S}^{\prime}$,

$$
\rho\left(\mathcal{S}, \mathcal{S}^{\prime}\right)=\log \left(\operatorname{diam} \mathcal{S} / \operatorname{diam} \mathcal{S}^{\prime}\right)
$$

(see, e.g., [Baker and Rumely 2010, §2.7]). In terms of $\rho$, the generalized Hsia kernel $\left[\mathcal{S}, \mathcal{S}^{\prime}\right]_{\text {can }}$ with respect to $\mathcal{S}_{\text {can }}$ is interpreted as a Gromov product

$$
\log \left[\mathcal{S}, \mathcal{S}^{\prime}\right]_{\text {can }}=-\rho\left(\mathcal{S}^{\prime \prime}, \mathcal{S}_{\text {can }}\right) \quad \text { on } \mathrm{H}^{1} \times \mathrm{H}^{1},
$$

where $\mathcal{S}^{\prime \prime}$ is the unique point in $\mathrm{H}^{1}$ lying between $\mathcal{S}$ and $\mathcal{S}^{\prime}$, between $\mathcal{S}^{\prime}$ and $\mathcal{S}_{\text {can }}$, and between $\mathcal{S}_{\text {can }}$ and $\mathcal{S}$ (see [Favre and Rivera-Letelier 2006, §3.4]). Similarly, for every $\mathcal{S}, \mathcal{S}^{\prime} \in \mathrm{A}^{1}$,

$$
\left|\mathcal{S}-\mathcal{S}^{\prime}\right|_{\infty}=\operatorname{diam} \mathcal{S}^{\prime \prime}
$$

where $\mathcal{S}^{\prime \prime}$ is the smallest point in $A^{1}$ satisfying both $\mathcal{S}^{\prime \prime} \succeq \mathcal{S}$ and $\mathcal{S}^{\prime \prime} \succeq \mathcal{S}^{\prime}$ with respect to the partial ordering $\succeq$ on $\mathrm{P}^{1}$.

For every $\epsilon>0$, a continuous mapping

$$
\pi_{\epsilon}: A^{1} \rightarrow A^{1}
$$

is defined by $\pi_{\epsilon}(\mathcal{S}):=\mathcal{S}^{\prime \prime}$ for every $\mathcal{S} \in \mathrm{A}^{1}$, where $\mathcal{S}^{\prime \prime} \in\left\{\mathcal{S} \in \mathrm{P}^{1}: \operatorname{diam} \mathcal{S} \in[\epsilon,+\infty)\right\}$ is the unique point between $\infty$ and $\mathcal{S}$ satisfying $\operatorname{diam} \mathcal{S}^{\prime \prime}=\max \{\epsilon, \operatorname{diam} \mathcal{S}\}$ (see [Favre and Rivera-Letelier 2006, §4.6] for details).

3.3. Regularization on $\mathbf{P}^{\mathbf{1}}$. When $K$ is archimedean, fix a nonnegative smooth decreasing function $\xi:[0, \infty) \rightarrow[0,1]$ such that supp $\xi \subset[0,1]$ and $\int_{0}^{\infty} \xi(x) \mathrm{d} x=1$, and set $\xi_{\epsilon}(x):=\xi(x / \epsilon) / \epsilon$ on $[0,+\infty)$ for each $\epsilon>0$. For every $z \in K$ and every 
$\epsilon>0$, the $\epsilon$-regularization $[z]_{\epsilon}$ of $\delta_{z}$ is the convolution $\xi_{\epsilon} * \delta_{z}$ on $\mathbb{P}^{1}$, i.e., for any continuous test function $\phi$ on $\mathbb{P}^{1}$,

$$
\left(\xi_{\epsilon} * \delta_{z}\right)(\phi)=\int_{0}^{\epsilon} \xi_{\epsilon}(r) \mathrm{d} r \int_{0}^{2 \pi} \phi\left(z+r e^{i \theta}\right) \frac{\mathrm{d} \theta}{2 \pi} .
$$

When $K$ is nonarchimedean, for every $z \in K$ and every $\epsilon>0$, the $\epsilon$-regularization $[z]_{\epsilon}$ of $\delta_{z}$ is defined by $[z]_{\epsilon}:=\left(\pi_{\epsilon}\right)_{*} \delta_{z}=\delta_{\pi_{\epsilon}(z)}$ on $\mathrm{P}^{1}$ [Favre and Rivera-Letelier 2006, p. 343]. In both cases, $[z]_{\epsilon}$ is a probability Radon measure on $\mathrm{P}^{1}$, the chordal potential $\mathrm{P}^{1} \ni \mathcal{S} \mapsto \int_{\mathrm{P} 1} \log \left[\mathcal{S}, \mathcal{S}^{\prime}\right]_{\text {can }} \mathrm{d}[z]_{\epsilon}\left(\mathcal{S}^{\prime}\right)$ of $[z]_{\epsilon}$ is a continuous function on $\mathrm{P}^{1}$, and for every $z, w \in K$ and every $\epsilon>0$, the estimate

$$
\int_{\mathrm{A}^{1} \times \mathrm{A}^{1}} \log \left|\mathcal{S}-\mathcal{S}^{\prime}\right|_{\infty} \mathrm{d}\left([z]_{\epsilon} \times[w]_{\epsilon}\right)\left(\mathcal{S}, \mathcal{S}^{\prime}\right) \geq \begin{cases}\log |z-w| & \text { if } z \neq w \\ C_{\mathrm{abs}}+\log \epsilon & \text { if } z=w\end{cases}
$$

holds, where $C_{\mathrm{abs}} \leq 0$ is an absolute constant and in fact $C_{\mathrm{abs}}=0$ for nonarchimedean $K$ [Favre and Rivera-Letelier 2006, Lemmes 2.10, 4.11, and their proofs].

Let us extend the $\epsilon$-regularization $[\cdot]_{\epsilon}$ and the estimate (3-6) to $\mathrm{P}^{1}$. Set $\iota(z):=$ $1 / z \in \operatorname{PGL}(2, K)$, which extends to an automorphism on $\mathrm{P}^{1}$ (see Fact 8.2), so that $\iota^{2}=\operatorname{Id}$ on $\mathrm{P}^{1}$ and $\left[\iota(\mathcal{S}), \iota\left(\mathcal{S}^{\prime}\right)\right]_{\mathrm{can}}=\left[\mathcal{S}, \mathcal{S}^{\prime}\right]_{\text {can }}\left(\operatorname{sod}\left(\iota(\mathcal{S}), \iota\left(\mathcal{S}^{\prime}\right)\right)=\mathrm{d}\left(\mathcal{S}, \mathcal{S}^{\prime}\right)\right)$ on $\mathrm{P}^{1} \times \mathrm{P}^{1}$. For every $\epsilon>0$, set $[\infty]_{\epsilon}:=\iota_{*}[0]_{\epsilon}$.

For every $z \in \mathbb{P}^{1}$ and every $\epsilon>0$, we have

$$
\operatorname{supp}[z]_{\epsilon} \subset\left\{\mathcal{S} \in \mathrm{P}^{1}: \mathrm{d}(\mathcal{S}, z) \leq \epsilon\right\},
$$

as follows immediately from the definitions of $\left|\mathcal{S}-\mathcal{S}^{\prime}\right|_{\infty}$ (and (3-5)), d, and $[z]_{\epsilon}$ when $z \in K$, and from (3-7) applied to $z=0$ and the invariance of $\mathrm{d}$ under $\iota$ when $z=\infty$. Moreover, for every $z \in K$ and every $\epsilon>0$,

$$
\sup _{\mathcal{S} \in \operatorname{supp}[z]_{\epsilon}}\left|\log [\mathcal{S}, \infty]_{\text {can }}-\log [z, \infty]\right| \leq \epsilon
$$

by a direct computation of $\log [\cdot, \infty]_{\mathrm{can}}-\log [z, \infty]$ on $K$, using that $\operatorname{supp}[z]_{\epsilon} \subset$ $\left\{\mathcal{S} \in \mathbb{P}^{1}:|\mathcal{S}-z|_{\infty} \leq \epsilon\right\}$ and the density of $K$ in $\mathrm{A}^{1}$.

Lemma 3.2. Let $g$ be a continuous weight on $\mathrm{P}^{1}$ having a modulus of continuity $\eta$ on $\left(\mathrm{P}^{1}, \mathrm{~d}\right)$. Then for every $\epsilon>0$ and every $z, w \in \mathbb{P}^{1}$,

$$
\begin{aligned}
\int_{\mathrm{P}^{1} \times \mathrm{P}^{1}} \Phi_{g} \mathrm{~d}\left([z]_{\epsilon} \times[w]_{\epsilon}\right) \\
\quad \geq \begin{cases}\Phi_{g}(z, w)-2 \epsilon-2 \eta(\epsilon) & \text { if } z \neq w, \\
C_{\mathrm{abs}}+\log \epsilon-2 \epsilon+2 \log [z, \infty]-2 \eta(\epsilon)-2 g(z) & \text { if } z=w \in K, \\
C_{\mathrm{abs}}+\log \epsilon-2 \epsilon-2 \eta(\epsilon)-2 g(\infty) & \text { if } z=w=\infty .\end{cases}
\end{aligned}
$$

Proof. Since $\Phi_{g}\left(\mathcal{S}, \mathcal{S}^{\prime}\right)=\log \left[\mathcal{S}, \mathcal{S}^{\prime}\right]_{\text {can }}-g(\mathcal{S})-g\left(\mathcal{S}^{\prime}\right)$ on $\mathrm{P}^{1} \times \mathrm{P}^{1}$, by (3-7), we can assume $g \equiv 0$ (and $\eta \equiv 0$ ) on $\mathrm{P}^{1}$ without loss of generality. For every $z, w \in K$, 
by the definition (3-2) of $\left|\mathcal{S}-\mathcal{S}^{\prime}\right|_{\infty}$ and (3-8),

$$
\begin{aligned}
\int_{\mathrm{P}^{1} \times \mathrm{P}^{1}} & \log \left[\mathcal{S}, \mathcal{S}^{\prime}\right]_{\operatorname{can}} \mathrm{d}\left([z]_{\epsilon} \times[w]_{\epsilon}\right)\left(\mathcal{S}, \mathcal{S}^{\prime}\right) \\
\quad \geq & \int_{\mathrm{A}^{1} \times \mathrm{A}^{1}} \log \left|\mathcal{S}-\mathcal{S}^{\prime}\right|_{\infty} \mathrm{d}\left([z]_{\epsilon} \times[w]_{\epsilon}\right)\left(\mathcal{S}, \mathcal{S}^{\prime}\right)-2 \epsilon+\log [z, \infty]+\log [w, \infty],
\end{aligned}
$$

which with the estimate (3-6) yields (3-9) (for $g \equiv \eta \equiv 0$ ) in this case. The estimate (3-9) (for $g \equiv \eta \equiv 0$ ) in the case $z=w=\infty$ follows from $[\infty]_{\epsilon}=\iota_{*}[0]_{\epsilon}$, $\left[\iota(\mathcal{S}), \iota\left(\mathcal{S}^{\prime}\right)\right]_{\mathrm{can}}=\left[\mathcal{S}, \mathcal{S}^{\prime}\right]_{\mathrm{can}}$, and the estimate (3-9) for $z=w=0$.

There remains the case that $z=\infty$ and $w \in K$ (so $z \neq w)$. If $K$ is nonarchimedean, then for every $w \in K$ and $\epsilon>0$, the equalities $[\infty]_{\epsilon}=\iota_{*}[0]_{\epsilon}$ and $\left[\iota(\mathcal{S}), \iota\left(\mathcal{S}^{\prime}\right)\right]_{\text {can }}=$ $\left[\mathcal{S}, \mathcal{S}^{\prime}\right]_{\text {can }}$, together with the interpretation (3-4) of $\left[\mathcal{S}, \mathcal{S}^{\prime}\right]_{\text {can }}$, yield

$$
\begin{aligned}
\int_{\mathrm{P}^{1} \times \mathrm{P}^{1}} & \log \left[\mathcal{S}, \mathcal{S}^{\prime}\right]_{\text {can }} \mathrm{d}\left([\infty]_{\epsilon} \times[w]_{\epsilon}\right)\left(\mathcal{S}, \mathcal{S}^{\prime}\right) \\
= & \int_{\mathrm{P}^{1} \times \mathrm{P}^{1}} \log \left[\mathcal{S}, \mathcal{S}^{\prime}\right]_{\mathrm{can}} \mathrm{d}\left([0]_{\epsilon} \times \iota_{*}[w]_{\epsilon}\right)\left(\mathcal{S}, \mathcal{S}^{\prime}\right)=\log \left[\pi_{\epsilon}(0), \iota\left(\pi_{\epsilon}(w)\right)\right]_{\mathrm{can}} \\
& \geq \log [0, \iota(w)]=\log [\infty, w] \geq \log [\infty, w]-2 \epsilon,
\end{aligned}
$$

which implies the estimate (3-9) (for $g \equiv \eta \equiv 0$ ) in the case $z=\infty$ and $w \in K$ when $K$ is nonarchimedean. If $K$ is archimedean, then for every $w \in K$ and every $r, r^{\prime}>0$, we have

$$
\begin{aligned}
\int_{0}^{2 \pi} & \frac{\mathrm{d} \phi}{2 \pi} \int_{0}^{2 \pi} \log \left|\left(0+r e^{i \theta}\right)-\frac{1}{w+r^{\prime} e^{i \phi}}\right| \frac{\mathrm{d} \theta}{2 \pi} \\
\quad & \int_{0}^{2 \pi} \max \left\{-\log \left|w+r^{\prime} e^{i \phi}\right|, \log r\right\} \frac{\mathrm{d} \phi}{2 \pi} \geq-\int_{0}^{2 \pi} \log \left|\left(w+r^{\prime} e^{i \phi}\right)-0\right| \frac{\mathrm{d} \phi}{2 \pi},
\end{aligned}
$$

so that for every $w \in K \cong \mathrm{A}^{1}$ and every $\epsilon>0$,

$$
\begin{aligned}
& \int_{\mathrm{A}^{1} \times \mathrm{A}^{1}} \log \left|\mathcal{S}-\mathcal{S}^{\prime}\right|_{\infty} \mathrm{d}\left([0]_{\epsilon} \times \iota_{*}[w]_{\epsilon}\right)\left(\mathcal{S}, \mathcal{S}^{\prime}\right) \\
& \quad=\int_{\mathrm{A}^{1} \times \mathrm{A}^{1}} \log \left|\mathcal{S}-\iota\left(\mathcal{S}^{\prime}\right)\right|_{\infty} \mathrm{d}\left([0]_{\epsilon} \times[w]_{\epsilon}\right)\left(\mathcal{S}, \mathcal{S}^{\prime}\right) \geq-\int_{\mathrm{A}^{1}} \log \left|\mathcal{S}^{\prime}-0\right|_{\infty} \mathrm{d}[w]_{\epsilon}\left(\mathcal{S}^{\prime}\right) .
\end{aligned}
$$

On the other hand, for every $w \in K$ and every $\epsilon>0$, by the definition (2-1) of the chordal metric $[z, w]$ on $\mathbb{P}^{1} \cong \mathrm{P}^{1}$ (and $\left.[0, \infty]=1\right)$,

$$
\begin{aligned}
\int_{\mathrm{P}^{1}} \log \left[\mathcal{S}^{\prime}, \infty\right]_{\mathrm{can}} \mathrm{d}\left(\iota_{*}[w]_{\epsilon}\right)\left(\mathcal{S}^{\prime}\right)=\int_{\mathrm{P}^{1}} \log \left[\mathcal{S}^{\prime}, 0\right]_{\mathrm{can}} \mathrm{d}[w]_{\epsilon}\left(\mathcal{S}^{\prime}\right) \\
=\int_{\mathrm{A}^{1}} \log \left|\mathcal{S}^{\prime}-0\right|_{\infty} \mathrm{d}[w]_{\epsilon}\left(\mathcal{S}^{\prime}\right)+\int_{\mathrm{P}^{1}} \log \left[\mathcal{S}^{\prime}, \infty\right]_{\mathrm{can}} \mathrm{d}[w]_{\epsilon}\left(\mathcal{S}^{\prime}\right) .
\end{aligned}
$$

From these computations and (3-8), for every $w \in K$ and every $\epsilon>0$, we get 


$$
\begin{aligned}
\int_{\mathrm{P}^{1} \times \mathrm{P}^{1}} \log \left[\mathcal{S}, \mathcal{S}^{\prime}\right]_{\mathrm{can}} \mathrm{d}( & {\left.[\infty]_{\epsilon} \times[w]_{\epsilon}\right)\left(\mathcal{S}, \mathcal{S}^{\prime}\right) } \\
& =\int_{\mathrm{P}^{1} \times \mathrm{P}^{1}} \log \left[\mathcal{S}, \mathcal{S}^{\prime}\right]_{\mathrm{can}} \mathrm{d}\left([0]_{\epsilon} \times \iota_{*}[w]_{\epsilon}\right)\left(\mathcal{S}, \mathcal{S}^{\prime}\right) \\
& \geq \int_{\mathrm{P}^{1}} \log [\mathcal{S}, \infty]_{\operatorname{can}} \mathrm{d}[0]_{\epsilon}(\mathcal{S})+\int_{\mathrm{P}^{1}} \log \left[\mathcal{S}^{\prime}, \infty\right]_{\operatorname{can}} \mathrm{d}[w]_{\epsilon}\left(\mathcal{S}^{\prime}\right) \\
& \geq \log [0, \infty]+\log [w, \infty]-2 \epsilon=\log [w, \infty]-2 \epsilon,
\end{aligned}
$$

which implies the estimate (3-9) (for $g \equiv \eta \equiv 0$ ) in the case $z=\infty$ and $w \in K$ when $K$ is archimedean.

\section{The negativity of regularized Fekete sums and a Cauchy-Schwarz inequality}

Let $K$ be an algebraically closed field that is complete with respect to a nontrivial absolute value $|\cdot|$. For every $\epsilon>0$ and every discrete measure $v$ on $\mathrm{P}^{1}=\mathrm{P}^{1}(K)$ whose support is in $\mathbb{P}^{1}=\mathbb{P}^{1}(K)$, the $\epsilon$-regularization of $v$ is

$$
v_{\epsilon}:=\sum_{w \in \operatorname{supp} v} v(\{w\})[w]_{\epsilon} \quad \text { on } \mathrm{P}^{1} .
$$

For every continuous weight $g$ on $\mathrm{P}^{1}$, let us call $\left(v_{\epsilon}, v_{\epsilon}\right)_{g}$ the $\epsilon$-regularized $g$-Fekete sum with respect to this $v$.

4.1. $C^{1}$-regularity and the Dirichlet norm. Recall the description of $\mathrm{P}^{1}$ given in Section 3.2. For nonarchimedean $K$, a function $\phi$ on $\mathrm{P}^{1}=\mathrm{P}^{1}(K)$ is in $C^{1}\left(\mathrm{P}^{1}\right)$ if

(i) $\phi$ is continuous on $\mathrm{P}^{1}$ and locally constant except for a union $\mathcal{T}$ of at most finitely many segments in $\mathrm{H}^{1}=\mathrm{H}^{1}(K)$, which are oriented by the partial ordering $\succeq$ on $\mathrm{P}^{1}$, and

(ii) the derivative $\phi^{\prime}$ with respect to the length parameter induced by the hyperbolic metric $\rho$ on each segment in $\mathcal{T}$ exists and is continuous on $\mathcal{T}$.

The Dirichlet norm of $\phi \in C^{1}\left(\mathrm{P}^{1}\right)$ is defined by $\langle\phi, \phi\rangle^{1 / 2}:=\left(\int_{\mathcal{T}}\left(\phi^{\prime}\right)^{2} \mathrm{~d} \rho\right)^{1 / 2}$, where $\mathrm{d} \rho$ is the 1-dimensional Hausdorff measure on $\mathrm{H}^{1}$ with respect to $\rho$ (for details, see [Favre and Rivera-Letelier 2006, §5.5]). When $K$ is archimedean, the $C^{1}$-regularity and the Dirichlet norm of a function $\phi$ on $\mathrm{P}^{1} \cong \mathbb{P}^{1}$ is defined with respect to the complex (or differentiable) structure of $\mathbb{P}^{1}$. For completeness, we include a proof of the following.

Proposition 4.1. Every $\phi$ in $C^{1}\left(\mathrm{P}^{1}\right)$ is Lipschitz continuous on $\left(\mathrm{P}^{1}, \mathrm{~d}\right)$.

Proof. When $K$ is archimedean, this is obvious. Suppose that $K$ is nonarchimedean and let $\phi \in C^{1}\left(\mathrm{P}^{1}\right)$. By definition, $\phi$ is locally constant on $\mathrm{P}^{1}$ except for a union 
$\mathcal{T}$ of at most finitely many segments in $\mathrm{H}^{1}$, and is Lipschitz continuous on $\mathcal{T}$ with respect to $\rho$. The set $\mathcal{T}$ is compact in $\left(\mathrm{H}^{1}, \rho\right)$, and for every $\mathcal{S}, \mathcal{S}^{\prime} \in \mathrm{H}^{1}$, by the definition (3-1) of d, (3-4), and (3-3), if $\mathcal{S}_{\text {can }} \succeq \mathcal{S} \succeq \mathcal{S}^{\prime}$, then

$$
\mathrm{d}\left(\mathcal{S}, \mathcal{S}^{\prime}\right)=\operatorname{diam} \mathcal{S}-\frac{\operatorname{diam} \mathcal{S}+\operatorname{diam} \mathcal{S}^{\prime}}{2}=\frac{\operatorname{diam} \mathcal{S}-\operatorname{diam} \mathcal{S}^{\prime}}{2} \geq \frac{\operatorname{diam} \mathcal{S}^{\prime}}{2} \rho\left(\mathcal{S}, \mathcal{S}^{\prime}\right),
$$

and similarly, if $\mathcal{S}_{\text {can }} \preceq \mathcal{S} \preceq \mathcal{S}^{\prime}$, then $\mathrm{d}\left(\mathcal{S}, \mathcal{S}^{\prime}\right) \geq \rho\left(\mathcal{S}, \mathcal{S}^{\prime}\right) /\left(2\right.$ diam $\left.\mathcal{S}^{\prime}\right)$. Hence we conclude that $\phi$ is also Lipschitz continuous on $\mathcal{T}$ with respect to $d$, and in turn on the whole $P^{1}$ with respect to $d$.

The Lipschitz constant of a Lipschitz continuous function $\phi$ on $\left(P^{1}, d\right)$ is denoted by $\operatorname{Lip}(\phi)$.

Remark 4.2. When $K$ is archimedean ( $\mathrm{P}^{1} \cong \mathbb{P}^{1}$ ), we have $\langle\phi, \phi\rangle^{1 / 2} \leq \operatorname{Lip}(\phi)$ for every $\phi \in C^{1}\left(\mathbb{P}^{1}\right)$. Moreover, every Lipschitz continuous function $\phi$ on $\left(\mathbb{P}^{1},[z, w]\right)$ is approximated by functions in $C^{1}\left(\mathbb{P}^{1}\right)$ in the Lipschitz norm.

4.2. The negativity of $\left(v_{\epsilon}, v_{\epsilon}\right)_{g}$ and a Cauchy-Schwarz inequality. For every Radon measure $\mu$ on $\mathrm{P}^{1}$ satisfying $\mu\left(\mathrm{P}^{1}\right)=0$, if the chordal potential of $\mu$, which is defined by $\mathcal{S} \mapsto \int_{\mathrm{P}^{1}} \log \left[\mathcal{S}, \mathcal{S}^{\prime}\right]_{\operatorname{can}} \mathrm{d} \mu\left(\mathcal{S}^{\prime}\right)$, is continuous on $\mathrm{P}^{1}$, then we have the positivity property $\int_{\mathrm{P}^{1} \times \mathrm{P}^{1}}\left(-\log \left|\mathcal{S}-\mathcal{S}^{\prime}\right|_{\infty}\right) \mathrm{d}(\mu \times \mu)\left(\mathcal{S}, \mathcal{S}^{\prime}\right) \geq 0$ (see [Favre and Rivera-Letelier 2006, §2.5 and §4.5]) and in fact the Cauchy-Schwarz inequality

$$
\left|\int_{\mathrm{P}^{1}} \phi \mathrm{d} \mu\right|^{2} \leq\langle\phi, \phi\rangle \cdot \int_{\mathrm{P}^{1} \times \mathrm{P}^{1}}\left(-\log \left|\mathcal{S}-\mathcal{S}^{\prime}\right|_{\infty}\right) \mathrm{d}(\mu \times \mu)\left(\mathcal{S}, \mathcal{S}^{\prime}\right)
$$

for every test function $\phi \in C^{1}\left(\mathrm{P}^{1}\right)$ (see [Favre and Rivera-Letelier 2006, (32) and (33)]).

In particular, for every $\epsilon>0$, every normalized weight $g$ on $\mathrm{P}^{1}$, every test function $\phi \in C^{1}\left(\mathrm{P}^{1}\right)$, and every discrete measure $v$ on $\mathrm{P}^{1}$ whose support is in $\mathbb{P}^{1}$, the computation

$$
\begin{aligned}
0 & \leq \int_{\mathrm{P}^{1} \times \mathrm{P}^{1}}\left(-\log \left|\mathcal{S}-\mathcal{S}^{\prime}\right|_{\infty}\right) \mathrm{d}\left(\left(v_{\epsilon}-\left(v\left(\mathrm{P}^{1}\right)\right) \mu^{g}\right) \times\left(v_{\epsilon}-\left(v\left(\mathrm{P}^{1}\right)\right) \mu^{g}\right)\right)\left(\mathcal{S}, \mathcal{S}^{\prime}\right) \\
& =\int_{\mathrm{P}^{1} \times \mathrm{P}^{1}}\left(-\Phi_{g}\right) \mathrm{d}\left(\left(v_{\epsilon}-\left(v\left(\mathrm{P}^{1}\right)\right) \mu^{g}\right) \times\left(v_{\epsilon}-\left(v\left(\mathrm{P}^{1}\right)\right) \mu^{g}\right)\right)=-\left(v_{\epsilon}, v_{\epsilon}\right)_{g}
\end{aligned}
$$

(recalling $U_{g, \mu^{g}} \equiv 0$ on $\mathrm{P}^{1}$ ) yields not only the negativity $\left(v_{\epsilon}, v_{\epsilon}\right)_{g} \leq 0$ but, with the Cauchy-Schwarz inequality (4-1) and the triangle inequality, also the estimate

$$
\begin{aligned}
\left|\int_{\mathrm{P}^{1}} \phi \mathrm{d}\left(v-v\left(\mathrm{P}^{1}\right) \mu^{g}\right)\right| & =\left|\int_{\mathrm{P}^{1}} \phi \mathrm{d}\left(\left(v-v_{\epsilon}\right)+\left(v_{\epsilon}-(\operatorname{deg} v) \mu^{g}\right)\right)\right| \\
& \leq(\operatorname{deg} v) \operatorname{Lip}(\phi) \epsilon+\langle\phi, \phi\rangle^{1 / 2} \cdot\left(-\left(v_{\epsilon}, v_{\epsilon}\right)_{g}\right)^{1 / 2} .
\end{aligned}
$$




\section{Computations of Fekete sums $(\mathcal{Z}, \mathcal{Z})_{g}$}

Let $k$ be a field. For a $k$-effective divisor $\mathcal{Z}$ on $\mathbb{P}^{1}(\bar{k})$, set

$$
D^{*}(\mathcal{Z} \mid \bar{k}):=\prod_{w \in \operatorname{supp} \mathcal{Z} \backslash\{\infty\}} \prod_{w^{\prime} \in \operatorname{supp} \mathcal{Z} \backslash\{w, \infty\}}\left(w-w^{\prime}\right)^{\left(\operatorname{ord}_{w} \mathcal{Z}\right)\left(\operatorname{ord}_{w^{\prime}} \mathcal{Z}\right)} \in \bar{k} \backslash\{0\},
$$

which is in fact in $k \backslash\{0\}$ by Theorem 7 if $\mathcal{Z}$ is on $\mathbb{P}^{1}\left(k_{s}\right)$. For every $P \in$ $\bigcup_{d \in \mathbb{N}} k\left[p_{0}, p_{1}\right]_{d}$, let $L(P(1, \cdot)) \in k \backslash\{0\}$ be the coefficient of the maximal degree term of $P(1, z) \in k[z]$ (appearing in Section 2.3).

Lemma 5.1. Let $k$ be a field. Let $\mathcal{Z}$ be a $k$-effective divisor on $\mathbb{P}^{1}(\bar{k})$ represented by $P \in \bigcup_{d \in \mathbb{N}} k\left[p_{0}, p_{1}\right]_{d}$, and let $\left(q_{j}^{P}\right)_{j=1}^{\operatorname{deg} P}$ be a sequence in $\bar{k}^{2} \backslash\{0\}$ giving a factorization (2-9) of $P$. For each $j \in\{1,2, \ldots, \operatorname{deg} P\}$, set $q_{j}^{P}=\left(\left(q_{j}^{P}\right)_{0},\left(q_{j}^{P}\right)_{0}\right)$ and $z_{j}:=\pi\left(q_{j}^{P}\right) \in \mathbb{P}^{1}(\bar{k})$. Suppose $\left(q_{j}^{P}\right)_{j=1}^{\operatorname{deg} P}$ is normalized with respect to a distinguished zero $w_{0} \in \mathbb{P}^{1}(\bar{k})$ of $P$ so that for each $j \in\{1,2, \ldots, \operatorname{deg} P\}$,

$$
\begin{cases}\left(q_{j}^{P}\right)_{0}=1 & \text { if } z_{j} \notin\left\{w_{0}, \infty\right\} \\ \left(q_{j}^{P}\right)_{1}=1 & \text { if } w_{0} \neq z_{j}=\infty .\end{cases}
$$

Then

$$
L(P(1, \cdot))=(-1)^{\operatorname{deg} P-\operatorname{deg}_{\infty} P} \cdot \begin{cases}\prod_{j: z_{j}=w_{0}}\left(q_{j}^{P}\right)_{0} & \text { if } w_{0} \neq \infty, \\ \prod_{j: z_{j}=w_{0}}\left(q_{j}^{P}\right)_{1} & \text { if } w_{0}=\infty,\end{cases}
$$

and

$$
\begin{aligned}
& \prod_{j=1}^{\operatorname{deg} P} \prod_{i: z_{i} \neq z_{j}}\left(q_{i}^{P} \wedge q_{j}^{P}\right) \\
& \quad=(-1)^{\operatorname{deg}_{\infty} P\left(\operatorname{deg} P-\operatorname{deg}_{\infty} P\right)} \cdot L(P(1, \cdot))^{2\left(\operatorname{deg} P-\operatorname{deg}_{w_{0}} P\right)} \cdot D^{*}(\mathcal{Z} \mid \bar{k}) .
\end{aligned}
$$

Proof. Without normalizing the sequence $\left(q_{j}^{P}\right)_{j=1}^{\operatorname{deg} P}$ we have, by direct computation,

$$
\begin{aligned}
& \prod_{j=1}^{\operatorname{deg} P} \prod_{\substack{i: z_{i} \neq z_{j} \\
=}}\left(q_{i}^{P} \wedge q_{j}^{P}\right) \\
& \left.=\prod_{\substack{j: z_{j}=\infty \\
i: z_{i} \neq \infty}}\left(\left(q_{i}^{P}\right)_{0}\left(q_{j}^{P}\right)_{1}\right) \cdot \prod_{\substack{j: z_{j} \neq \infty \\
i: z_{i}=\infty}}\left(-\left(q_{i}^{P}\right)_{1}\left(q_{j}^{P}\right)_{0}\right)\right) \cdot \prod_{\substack{j: z_{j} \neq \infty \\
i: z_{i} \notin\left\{z_{j}, \infty\right\}}}\left(\left(q_{i}^{P}\right)_{0}\left(q_{j}^{P}\right)_{0}\left(z_{j}-z_{i}\right)\right) \\
& =(-1)^{\operatorname{deg}_{\infty} P\left(\operatorname{deg} P-\operatorname{deg}_{\infty} P\right)} \cdot\left(\prod_{j: z_{j}=\infty}\left(\left(q_{j}^{P}\right)_{1}^{\operatorname{deg} P-\operatorname{deg}_{\infty} P} \cdot \prod_{i: z_{i} \neq \infty}\left(q_{i}^{P}\right)_{0}\right)\right)^{2} \\
& \cdot\left(\prod_{j: z_{j} \neq \infty}\left(\left(q_{j}^{P}\right)_{0}^{\operatorname{deg} P-\operatorname{deg}_{\infty} P-\operatorname{deg}_{z_{j}} P} \cdot \prod_{i: z_{i} \notin\left\{z_{j}, \infty\right\}}\left(q_{i}^{P}\right)_{0}\right)\right) \cdot D^{*}(\mathcal{Z} \mid \bar{k}) .
\end{aligned}
$$

Let us normalize $\left(q_{j}^{P}\right)$ so that the normalization (5-1) holds with respect to a 
distinguished zero $w_{0} \in \mathbb{P}^{1}(\bar{k})$ of $P$. Then (5-2) follows from

$$
L(P(1, \cdot))=(-1)^{\operatorname{deg} P-\operatorname{deg}_{\infty} P} \cdot\left(\prod_{j: z_{j}=\infty}\left(q_{j}^{P}\right)_{1}\right)\left(\prod_{j: z_{j} \neq \infty}\left(q_{j}^{P}\right)_{0}\right)
$$

and the normalization (5-1).

Let us show (5-3). If $w_{0}=\infty$, then under the normalization (5-1), the equality (5-4) yields

$$
\begin{aligned}
& \prod_{j=1}^{\operatorname{deg} P} \prod_{i: z_{i} \neq z_{j}}\left(q_{i}^{P} \wedge q_{j}^{P}\right) \\
& \quad=(-1)^{\operatorname{deg}_{\infty} P\left(\operatorname{deg} P-\operatorname{deg}_{\infty} P\right)} \cdot\left(\prod_{j: z_{j}=\infty}\left(q_{j}^{P}\right)_{1}\right)^{2\left(\operatorname{deg} P-\operatorname{deg}_{\infty} P\right)} \cdot 1 \cdot D^{*}(\mathcal{Z} \mid \bar{k}),
\end{aligned}
$$

which with (5-2) implies (5-3) when $w_{0}=\infty$. If $w_{0} \neq \infty$, then under the normalization (5-1), the equality (5-4) yields

$$
\begin{aligned}
\prod_{j=1}^{\operatorname{deg} P} \prod_{i: z_{i} \neq z_{j}}\left(q_{i}^{P} \wedge q_{j}^{P}\right) & \\
=(-1)^{\operatorname{deg}_{\infty} P\left(\operatorname{deg} P-\operatorname{deg}_{\infty} P\right)} & \cdot\left(\prod_{i: z_{i}=w_{0}}\left(q_{i}^{P}\right)_{0}\right)^{2 \operatorname{deg}_{\infty} P} \\
& \cdot\left(\prod_{j: z_{j}=w_{0}}\left(\left(q_{j}^{P}\right)_{0}^{\operatorname{deg} P-\operatorname{deg}_{\infty} P-\operatorname{deg}_{z_{j}} P} \cdot 1\right)\right) \\
& \cdot\left(\prod_{j: z_{j} \notin\left\{w_{0}, \infty\right\}}\left(1 \cdot \prod_{i: z_{i}=w_{0}}\left(q_{i}^{P}\right)_{0}\right)\right) \cdot D^{*}(\mathcal{Z} \mid \bar{k}) \\
=(-1)^{\operatorname{deg}_{\infty} P\left(\operatorname{deg} P-\operatorname{deg}_{\infty} P\right)} & \cdot\left(\prod_{i: z_{i}=w_{0}}\left(q_{i}^{P}\right)_{0}\right)^{2 \operatorname{deg}_{\infty} P+2\left(\operatorname{deg} P-\operatorname{deg}_{\infty} P-\operatorname{deg}_{w_{0}} P\right)} \cdot D^{*}(\mathcal{Z} \mid \bar{k}),
\end{aligned}
$$

which with (5-2) implies (5-3) when $w_{0} \neq \infty$.

Lemma 5.2 (local computation). Let $k$ be a field and $K$ an algebraic and metric augmentation of $k$ (see Section 2.2). For every continuous weight $g$ on $\mathrm{P}^{1}=\mathrm{P}^{1}(K)$ and every $k$-effective divisor $\mathcal{Z}$ on $\mathbb{P}^{1}(\bar{k})$ represented by a homogeneous polynomial $P \in \bigcup_{d \in \mathbb{N}} k\left[p_{0}, p_{1}\right]_{d}$, we have

$$
\begin{aligned}
(\mathcal{Z}, \mathcal{Z})_{g}+ & 2 \cdot \sum_{w \in \operatorname{supp} \mathcal{Z} \backslash\{\infty\}}\left(\operatorname{ord}_{w} \mathcal{Z}\right)^{2} \log [w, \infty]-2 \cdot \sum_{w \in \operatorname{supp} \mathcal{Z}}\left(\operatorname{ord}_{w} \mathcal{Z}\right)^{2} g(w) \\
& =2(\operatorname{deg} \mathcal{Z}) \log |L(P(1, \cdot))|+\log \left|D^{*}(\mathcal{Z} \mid \bar{k})\right|-2(\operatorname{deg} \mathcal{Z}) M_{g}(P) .
\end{aligned}
$$

Proof. Let $\mathcal{Z}$ and $P$ be as in the statement and let $\left(q_{j}^{P}\right)_{j=1}^{\operatorname{deg} P}$ be a sequence in $\bar{k}^{2} \backslash\{0\}$ giving a factorization (2-9) of $P$ and satisfying the normalization (5-1) with 
respect to a distinguished zero $w_{0} \in \mathbb{P}^{1}(\bar{k})$ of $P$. Set $z_{j}:=\pi\left(q_{j}^{P}\right) \in \mathbb{P}^{1}(\bar{k})$ for each $j \in\{1,2, \ldots, \operatorname{deg} P\}$. Since by definition

$$
\Phi_{g}\left(z, z^{\prime}\right)=\log \left[z, z^{\prime}\right]-g(z)-g\left(z^{\prime}\right)
$$

on $\mathbb{P}^{1}(K) \times \mathbb{P}^{1}(K)$, we have

$$
(\mathcal{Z}, \mathcal{Z})_{g}=\log \left(\prod_{j=1}^{\operatorname{deg} P} \prod_{i: z_{i} \neq z_{j}}\left|q_{i}^{P} \wedge q_{j}^{P}\right|\right)-2 \cdot \sum_{j=1}^{\operatorname{deg} P} \sum_{i: z_{i} \neq z_{j}}\left(g\left(z_{i}\right)+\log \left\|q_{i}^{P}\right\|\right) ;
$$

by $(5-3)$,

$\log \left(\prod_{j=1}^{\operatorname{deg} P} \prod_{i: z_{i} \neq z_{j}}\left|q_{i}^{P} \wedge q_{j}^{P}\right|\right)=2\left(\operatorname{deg} P-\operatorname{deg}_{w_{0}} P\right) \log |L(P(1, \cdot))|+\log \left|D^{*}(\mathcal{Z} \mid \bar{k})\right|$,

and we also have

$\sum_{j=1}^{\operatorname{deg} P} \sum_{i: z_{i} \neq z_{j}}\left(g\left(z_{i}\right)+\log \left\|q_{i}^{P}\right\|\right)$

$$
\begin{aligned}
& =\sum_{j=1}^{\operatorname{deg} P} \sum_{i=1}^{\operatorname{deg} P}\left(g\left(z_{i}\right)+\log \left\|q_{i}^{P}\right\|\right)-\sum_{j=1}^{\operatorname{deg} P} \sum_{i: z_{i}=z_{j}}\left(g\left(z_{i}\right)+\log \left\|q_{i}^{P}\right\|\right) \\
& =(\operatorname{deg} P) M_{g}(P)-\sum_{j=1}^{\operatorname{deg} P}\left(\operatorname{deg}_{z_{j}} P\right) g\left(z_{j}\right)-\sum_{j=1}^{\operatorname{deg} P} \sum_{i: z_{i}=z_{j}} \log \left\|q_{i}^{P}\right\|,
\end{aligned}
$$

where the final equality is by the definition (2-10) of $M_{g}(P)$. Hence

$(\mathcal{Z}, \mathcal{Z})_{g}=2(\operatorname{deg} P) \log |L(P(1, \cdot))|+\log \left|D^{*}(\mathcal{Z} \mid \bar{k})\right|-2(\operatorname{deg} P) M_{g}(P)$

$+2 \sum_{w \in \text { supp } \mathcal{Z}}\left(\operatorname{ord}_{w} \mathcal{Z}\right)^{2} g(w)-2\left(\left(\operatorname{deg}_{w_{0}} P\right) \log |L(P(1, \cdot))|-\sum_{j=1}^{\operatorname{deg} P} \sum_{i: z_{i}=z_{j}} \log \left\|q_{i}^{P}\right\|\right)$.

For each $j \in\{1,2, \ldots, \operatorname{deg} P\}$, also set $q_{j}^{P}=\left(\left(q_{j}^{P}\right)_{0},\left(q_{j}^{P}\right)_{0}\right)$. If $\infty \notin \operatorname{supp} \mathcal{Z}$, then $w_{0} \neq \infty$, and by the normalization (5-1) and the equality (5-2),

$$
\begin{aligned}
\left(\operatorname{deg}_{w_{0}} P\right) \log |L(P(1, \cdot))|-\sum_{j=1}^{\operatorname{deg} P} \sum_{i: z_{i}=z_{j}} \log \left\|q_{i}^{P}\right\| \\
=-\sum_{j=1} \sum_{i: z_{i}=z_{j}}\left(\log \left\|q_{i}^{P}\right\|-\log \left|\left(q_{i}^{P}\right)_{0}\right|\right)=\sum_{j=1}^{\operatorname{deg} P} \sum_{i: z_{i}=z_{j}} \log \left[z_{i}, \infty\right] \\
=\sum_{w \in \operatorname{supp} \mathcal{Z}}\left(\operatorname{ord}_{w} \mathcal{Z}\right)^{2} \log [w, \infty]=\sum_{w \in \operatorname{supp} \mathcal{Z} \backslash\{\infty\}}\left(\operatorname{ord}_{w} \mathcal{Z}\right)^{2} \log [w, \infty] .
\end{aligned}
$$


If $\infty \in \operatorname{supp} \mathcal{Z}$, then we can set $w_{0}=\infty$, and by the normalization (5-1) and the equality $(5-2)$ (and $q_{i}^{P}=\left(q_{i}^{P}\right)_{1} \cdot(0,1)$ when $\left.z_{i}=\infty\right)$,

$$
\begin{gathered}
\left(\operatorname{deg}_{w_{0}} P\right) \log |L(P(1, \cdot))|-\sum_{j=1}^{\operatorname{deg} P} \sum_{i: z_{i}=z_{j}} \log \left\|q_{i}^{P}\right\| \\
=-\sum_{j: z_{j}=\infty} \sum_{i: z_{i}=z_{j}}\left(\log \left\|q_{i}^{P}\right\|-\log \left|\left(q_{i}^{P}\right)_{1}\right|\right)-\sum_{j: z_{j} \neq \infty} \sum_{i: z_{i}=z_{j}}\left(\log \left\|q_{i}^{P}\right\|-\log \left|\left(q_{i}^{P}\right)_{0}\right|\right) \\
=\sum_{j: z_{j} \neq \infty} \sum_{i: z_{i}=z_{j}} \log \left[z_{i}, \infty\right]=\sum_{w \in \operatorname{supp} \mathcal{Z} \backslash\{\infty\}}\left(\operatorname{ord}_{w} \mathcal{Z}\right)^{2} \log [w, \infty] .
\end{gathered}
$$

This completes the proof.

Lemma 5.3 (global computation). Let $k$ be a product formula field and $k_{s}$ the separable closure of $k$ in $\bar{k}$. Then for every adelic continuous weight $g=\left\{g_{v}: v \in M_{k}\right\}$ and every $k$-effective divisor $\mathcal{Z}$ on $\mathbb{P}^{1}\left(k_{s}\right)$,

$$
\begin{aligned}
\sum_{v \in M_{k}} N_{v}\left((\mathcal{Z}, \mathcal{Z})_{g_{v}}\right. & \left.+2 \sum_{w \in \operatorname{supp} \mathcal{Z} \backslash\{\infty\}}\left(\operatorname{ord}_{w} \mathcal{Z}\right)^{2} \log [w, \infty]_{v}\right) \\
& =-2(\operatorname{deg} \mathcal{Z})^{2} h_{g}(\mathcal{Z})+2 \sum_{v \in M_{k}} N_{v} \sum_{w \in \operatorname{supp} \mathcal{Z}}\left(\operatorname{ord}_{w} \mathcal{Z}\right)^{2} g_{v}(w) .
\end{aligned}
$$

Proof. Let $P \in \bigcup_{d \in \mathbb{N}} k\left[p_{0}, p_{1}\right]_{d}$ be a representative of $\mathcal{Z}$. Summing up the product of $N_{v}$ and (5-5) (for this $P$ ) over all $v \in M_{k}$, we have

$$
\begin{array}{r}
\sum_{v \in M_{k}} N_{v}\left((\mathcal{Z}, \mathcal{Z})_{g_{v}}+2 \sum_{w \in \operatorname{supp} \mathcal{Z} \backslash\{\infty\}}\left(\operatorname{ord}_{w} \mathcal{Z}\right)^{2} \log [w, \infty]_{v}-2 \sum_{w \in \operatorname{supp} \mathcal{Z}}\left(\operatorname{ord}_{w} \mathcal{Z}\right)^{2} g_{v}(w)\right) \\
=-2(\operatorname{deg} \mathcal{Z})^{2} h_{g}(\mathcal{Z})
\end{array}
$$

by the product formula (PF) (since $L(P(1, \cdot)) \in k \backslash\{0\}$ and, under the assumption that $\mathcal{Z}$ is on $\left.\mathbb{P}^{1}\left(k_{s}\right), D^{*}(\mathcal{Z} \mid \bar{k}) \in k \backslash\{0\}\right)$ and the definition $(1-1)$ of $h_{g}(\mathcal{Z})$.

\section{Estimates of regularized Fekete sums $\left(\mathcal{Z}_{\epsilon}, \mathcal{Z}_{\epsilon}\right)_{g}$}

6.1. Local estimate. Let $k$ be a field and $K$ an algebraic and metric augmentation of $k$. Let $\mathcal{Z}$ be a $k$-effective divisor on $\mathbb{P}^{1}(\bar{k})$, which we regard as the Radon measure

$$
\sum_{w \in \operatorname{supp} \mathcal{Z}}\left(\operatorname{ord}_{w} \mathcal{Z}\right) \delta_{w}
$$

on $\mathrm{P}^{1}=\mathrm{P}^{1}(K)$, and let $g$ be a continuous weight on $\mathrm{P}^{1}$ such that $g$ is a $1 / \kappa$-Hölder continuous function on $\left(\mathrm{P}^{1}, \mathrm{~d}\right)$ for some $\kappa \geq 1$ having the $1 / \kappa$-Hölder constant $C(g) \geq 0$. 
Lemma 6.1. For every $\epsilon>0$,

$$
\begin{array}{r}
\left(\mathcal{Z}_{\epsilon}, \mathcal{Z}_{\epsilon}\right)_{g} \geq(\mathcal{Z}, \mathcal{Z})_{g}+2 \sum_{w \in \operatorname{supp} \mathcal{Z} \backslash\{\infty\}}\left(\operatorname{ord}_{w} \mathcal{Z}\right)^{2} \log [w, \infty]-2 \sum_{w \in \operatorname{supp} \mathcal{Z}}\left(\operatorname{ord}_{w} \mathcal{Z}\right)^{2} g(w) \\
+\left(C_{\text {abs }}+\log \epsilon\right) \cdot(\mathcal{Z} \times \mathcal{Z})\left(\operatorname{diag}_{\mathbb{P} 1(\bar{k})}\right)-2(\operatorname{deg} \mathcal{Z})^{2}\left(\epsilon+C(g) \epsilon^{1 / \kappa}\right) .
\end{array}
$$

Proof. Set $\eta(\epsilon)=C(g) \epsilon^{1 / \kappa}$. For every $\epsilon>0$, using (3-9),

$$
\begin{aligned}
& \left(\mathcal{Z}_{\epsilon}, \mathcal{Z}_{\epsilon}\right)_{g}-(\mathcal{Z}, \mathcal{Z})_{g} \\
& =\int_{\mathrm{P}^{1} \times \mathrm{P}^{1}} \Phi_{g} \mathrm{~d}\left(\mathcal{Z}_{\epsilon} \times \mathcal{Z}_{\epsilon}\right)-\int_{\mathrm{P}^{1} \times \mathrm{P}^{1} \backslash \operatorname{diag}_{\mathbb{P}^{1}(K)}} \Phi_{g} \mathrm{~d}(\mathcal{Z} \times \mathcal{Z}) \\
& =\sum_{w \in \operatorname{supp} \mathcal{Z}}\left(\operatorname{ord}_{w} \mathcal{Z}\right)^{2} \int_{\mathrm{P}^{1} \times \mathrm{P}^{1}} \Phi_{g} \mathrm{~d}\left([w]_{\epsilon} \times[w]_{\epsilon}\right) \\
& +\sum_{(z, w) \in \mathbb{P}^{1} \times \mathbb{P}^{1} \backslash \operatorname{diag}_{\mathbb{P}^{1}}}\left(\int_{\mathbb{P}^{1} \times \mathbb{P}^{1}} \Phi_{g}\left(\mathcal{S}, \mathcal{S}^{\prime}\right) \mathrm{d}\left([z]_{\epsilon} \times[w]_{\epsilon}\right)\left(\mathcal{S}, \mathcal{S}^{\prime}\right)-\Phi_{g}(z, w)\right) \\
& \geq \sum_{w \in \operatorname{supp} \mathcal{Z} \backslash\{\infty\}}\left(\operatorname{ord}_{w} \mathcal{Z}\right)^{2}\left(C_{\text {abs }}+\log \epsilon-2 \epsilon+2 \log [w, \infty]-2 \eta(\epsilon)-2 g(w)\right) \\
& +(\mathcal{Z}(\{\infty\}))^{2}\left(C_{\mathrm{abs}}+\log \epsilon-2 \epsilon-2 \eta(\epsilon)-2 g(\infty)\right) \\
& +\left((\operatorname{deg} \mathcal{Z})^{2}-(\mathcal{Z} \times \mathcal{Z})\left(\operatorname{diag}_{\mathbb{P}^{1}(\bar{k})}\right)\right)(-2 \epsilon-2 \eta(\epsilon)) \\
& =\left((\mathcal{Z} \times \mathcal{Z})\left(\operatorname{diag}_{\mathbb{P}^{1}(\bar{k})}\right)\right)\left(C_{\mathrm{abs}}+\log \epsilon-2 \epsilon-2 \eta(\epsilon)\right) \\
& +2 \sum_{w \in \operatorname{supp} \mathcal{Z} \backslash\{\infty\}}\left(\operatorname{ord}_{w} \mathcal{Z}\right)^{2} \log [w, \infty]-2 \sum_{w \in \operatorname{supp} \mathcal{Z}}\left(\operatorname{ord}_{w} \mathcal{Z}\right)^{2} g(w) \\
& +\left((\operatorname{deg} \mathcal{Z})^{2}-(\mathcal{Z} \times \mathcal{Z})\left(\operatorname{diag}_{\mathbb{P}^{1}(\bar{k})}\right)\right)(-2 \epsilon-2 \eta(\epsilon)),
\end{aligned}
$$

which completes the proof.

6.2. Global estimate. Let $k$ be a product formula field, and $\mathcal{Z}$ a $k$-effective divisor on $\mathbb{P}^{1}\left(k_{s}\right)$. Let $g=\left\{g_{v}: v \in M_{k}\right\}$ be a placewise Hölder continuous adelic normalized weight, so for every $v \in M_{k}, g_{v}$ is a normalized weight on $\mathrm{P}^{1}\left(\mathbb{C}_{v}\right)$ and is a $1 / \kappa_{v}$ Hölder continuous function on $\left(\mathrm{P}^{1}\left(\mathbb{C}_{v}\right), \mathrm{d}_{v}\right)$ for some $\kappa_{v} \geq 1$ having the $1 / \kappa_{v}$-Hölder constant $C\left(g_{v}\right) \geq 0$.

Lemma 6.2. For every $v_{0} \in M_{k}$ and every $\epsilon>0$,

$$
\begin{array}{r}
N_{v_{0}}\left(\mathcal{Z}_{\epsilon}, \mathcal{Z}_{\epsilon}\right)_{g_{v_{0}}} \geq-2(\operatorname{deg} \mathcal{Z})^{2} h_{g}(\mathcal{Z})+\left(C_{\mathrm{abs}}+\log \epsilon\right) \cdot(\mathcal{Z} \times \mathcal{Z})\left(\operatorname{diag}_{\mathbb{P l}}\left(k_{s}\right)\right) \cdot \sum_{v \in E_{g} \cup\left\{v_{0}\right\}} N_{v} \\
-2(\operatorname{deg} \mathcal{Z})^{2} \sum_{v \in E_{g} \cup\left\{v_{0}\right\}} N_{v}\left(\epsilon+C\left(g_{v}\right) \epsilon^{1 / \kappa_{v_{0}}}\right) .
\end{array}
$$

Proof. Fix $v_{0} \in M_{k}$. We use, for every $v \in M_{k}$, the notation 


$$
W_{v}:=(\mathcal{Z}, \mathcal{Z})_{g_{v}}+2 \sum_{w \in \operatorname{supp} \mathcal{Z} \backslash\{\infty\}}\left(\operatorname{ord}_{w} \mathcal{Z}\right)^{2} \log [w, \infty]_{v}-2 \sum_{w \in \operatorname{supp} \mathcal{Z}}\left(\operatorname{ord}_{w} \mathcal{Z}\right)^{2} g_{v}(w)
$$

Since $\left(\mathcal{Z}_{\epsilon}, \mathcal{Z}_{\epsilon}\right)_{g_{v}} \leq 0$ for every $\epsilon>0$ and every $v \in M_{k}$ (see Section 4.2), using also Lemma 6.1, we have

$$
\begin{aligned}
N_{v_{0}}\left(\mathcal{Z}_{\epsilon}, \mathcal{Z}_{\epsilon}\right)_{g_{0}} & \geq \sum_{v \in E_{g} \cup\left\{v_{0}\right\}} N_{v}\left(\mathcal{Z}_{\epsilon}, \mathcal{Z}_{\epsilon}\right)_{v_{0}} \\
\geq & \sum_{v \in E_{g} \cup\left\{v_{0}\right\}} N_{v} W_{v}+\left(C_{\text {abs }}+\log \epsilon\right) \cdot(\mathcal{Z} \times \mathcal{Z})\left(\operatorname{diag}_{\mathbb{P}^{1}\left(k_{s}\right)}\right) \cdot \sum_{v \in E_{g} \cup\left\{v_{0}\right\}} N_{v} \\
& -2(\operatorname{deg} \mathcal{Z})^{2} \sum_{v \in E_{g} \cup\left\{v_{0}\right\}} N_{v}\left(\epsilon+C\left(g_{v}\right) \epsilon^{1 / \kappa_{v_{0}}}\right) .
\end{aligned}
$$

Moreover, since for every $v \in M_{k} \backslash E_{g}, g_{v} \equiv 0$ on $\mathrm{P}^{1}\left(\mathbb{C}_{v}\right)$ and $(\mathcal{Z}, \mathcal{Z})_{g_{v}} \leq 0$, using also (5-6), we have

$$
\sum_{v \in E_{g} \cup\left\{v_{0}\right\}} N_{v} W_{v} \geq \sum_{v \in M_{k}} N_{v} W_{v}=-2(\operatorname{deg} \mathcal{Z})^{2} h_{g}(\mathcal{Z}),
$$

which completes the proof.

\section{Proofs of Theorems 1 and 2}

Proof of Theorem 1. Fix $v_{0} \in M_{k}$. For every $v \in M_{k}, g_{v}$ is a $1 / \kappa_{v}$-Hölder continuous function on $\left(\mathrm{P}^{1}\left(\mathbb{C}_{v}\right), \mathrm{d}_{v}\right)$ for some $\kappa_{v} \geq 1$ having the $1 / \kappa_{v}$-Hölder constant $C\left(g_{v}\right) \geq 0$. Set $\epsilon=1 /(\operatorname{deg} \mathcal{Z})^{2 \kappa_{v_{0}}}$. For every test function $\phi \in C^{1}\left(\mathrm{P}^{1}\left(\mathbb{C}_{v_{0}}\right)\right)$, by (4-2) and Lemma 6.2,

$$
\begin{aligned}
\left|\int_{\mathrm{P}^{1}\left(\mathbb{C}_{v_{0}}\right)} \phi \mathrm{d}\left(\frac{\mathcal{Z}}{\operatorname{deg} \mathcal{Z}}-\mu_{v_{0}}^{g}\right)\right| \leq \frac{\operatorname{Lip}(\phi)_{v_{0}}}{(\operatorname{deg} \mathcal{Z})^{2 \kappa_{0}}}+\frac{\langle\phi, \phi\rangle_{v_{0}}^{1 / 2}}{N_{v_{0}}^{1 / 2}} \\
\cdot\left(2 \cdot h_{g}(\mathcal{Z})+\left(-C_{\mathrm{abs}}+2 \kappa_{v_{0}} \log \operatorname{deg} \mathcal{Z}\right) \cdot \frac{(\mathcal{Z} \times \mathcal{Z})\left(\operatorname{diag}_{\mathbb{P}^{1}\left(k_{s}\right)}\right)}{(\operatorname{deg} \mathcal{Z})^{2}} \cdot \sum_{v \in E_{g} \cup\left\{v_{0}\right\}} N_{v}\right. \\
\left.+2 \sum_{v \in E_{g} \cup\left\{v_{0}\right\}} N_{v}\left(\frac{1}{(\operatorname{deg} \mathcal{Z})^{2 \kappa_{0}}}+\frac{C\left(g_{v}\right)}{(\operatorname{deg} \mathcal{Z})^{2}}\right)\right)^{1 / 2},
\end{aligned}
$$

which completes the proof.

Proof of Theorem 2. Fix $v_{0} \in M_{k}$. For every $n \in \mathbb{N}$, we have $\left(\mathcal{Z}_{n}, \mathcal{Z}_{n}\right)_{g_{v}} \leq 0$ if $v \in M_{k} \backslash E_{g}$. Hence by (2-8), (5-6), and the assumption that $V_{g_{v}}=0$ for every 
$v \in M_{k}$, we obtain

$$
\begin{aligned}
& N_{v_{0}} \frac{\left(\mathcal{Z}_{n}, \mathcal{Z}_{n}\right)_{g_{0}}}{\left(\operatorname{deg} \mathcal{Z}_{n}\right)^{2}}+\# E_{g} \cdot o(1) \geq \sum_{v \in M_{k}} N_{v} \frac{\left(\mathcal{Z}_{n}, \mathcal{Z}_{n}\right)_{g_{v}}}{\left(\operatorname{deg} \mathcal{Z}_{n}\right)^{2}} \\
& \geq-2 \cdot h_{g}\left(\mathcal{Z}_{n}\right)-2 \frac{\left(\mathcal{Z}_{n} \times \mathcal{Z}_{n}\right)\left(\operatorname{diag}_{\mathbb{P}^{1}\left(k_{s}\right)}\right)}{\left(\operatorname{deg} \mathcal{Z}_{n}\right)^{2}} \sum_{v \in E_{g}} N_{v} \sup _{\mathrm{P}^{1}\left(\mathbb{C}_{v}\right)}\left|g_{v}\right| \quad \text { as } n \rightarrow \infty ;
\end{aligned}
$$

thus, under the assumption that $\left(\mathcal{Z}_{n}\right)$ has both small diagonals and small $g$-heights, we have $\liminf _{n \rightarrow \infty}\left(\mathcal{Z}_{n}, \mathcal{Z}_{n}\right)_{g_{v_{0}}} /\left(\operatorname{deg} \mathcal{Z}_{n}\right)^{2} \geq 0=V_{g_{v_{0}}}$. Hence (2-7) holds for $g_{v_{0}}$ and $\left(\mathcal{Z}_{n}\right)$, and the proof is complete.

\section{Nonarchimedean and complex dynamics}

Fact 8.1. Let $k$ be a field. For a rational function $\phi \in k(z)$, we call

$$
F_{\phi}=\left(\left(F_{\phi}\right)_{0},\left(F_{\phi}\right)_{1}\right) \in \bigcup_{d \in \mathbb{N} \cup\{0\}}\left(k\left[p_{0}, p_{1}\right]_{d} \times k\left[p_{0}, p_{1}\right]_{d}\right)
$$

a lift of $\phi$ if $\pi \circ F_{\phi}=\phi \circ \pi$ on $k^{2} \backslash\{0\}$ and, in addition, $F_{\phi}^{-1}(0)=\{0\}$ when $\operatorname{deg} \phi>0$. The latter nondegeneracy condition is equivalent to the nonvanishing of $\operatorname{Res}\left(F_{\phi}\right):=$ $\operatorname{Res}\left(\left(F_{\phi}\right)_{0},\left(F_{\phi}\right)_{1}\right)$; for the definition of the homogeneous resultant $\operatorname{Res}(P, Q) \in k$ for $P, Q \in \bigcup_{d \in \mathbb{N} \cup\{0\}} k\left[p_{0}, p_{1}\right]_{d}$, see, e.g., [Silverman 2007, §2.4]. Such a lift $F_{\phi}$ of $\phi$ is unique up to multiplication in $k^{*}$, and is in fact in $k\left[p_{0}, p_{1}\right]_{\operatorname{deg} \phi} \times k\left[p_{0}, p_{1}\right]_{\operatorname{deg} \phi}$.

Let $K$ be an algebraically closed field that is complete with respect to a nontrivial absolute value $|\cdot|$.

8.1. The dynamical Green function $g_{f}$ on $\mathbf{P}^{1}$. For the foundation of a potentialtheoretical study of dynamics on the Berkovich projective line, see [Baker and Rumely 2010; Favre and Rivera-Letelier 2010] for nonarchimedean $K$ and, e.g., [Berteloot and Mayer 2001, §VIII] for archimedean $K(\cong \mathbb{C})$.

Fact 8.2. Let $\phi \in K(z)$ be a rational function of degree $d_{0} \in \mathbb{N} \cup\{0\}$. The action of $\phi$ on $\mathbb{P}^{1}=\mathbb{P}^{1}(K)$ uniquely extends to a continuous endomorphism on $\mathrm{P}^{1}=\mathrm{P}^{1}(K)$. When $d_{0}>0$, the extended $\phi$ is surjective, open, and discrete and preserves $\mathbb{P}^{1}$ and $\mathrm{H}^{1}=\mathrm{H}^{1}(K)$, the local degree function $z \mapsto \operatorname{deg}_{z} \phi$ on $\mathbb{P}^{1}$ also canonically extends to $\mathrm{P}^{1}$, and the (mapping) degree of the extended $\phi: \mathrm{P}^{1} \rightarrow \mathrm{P}^{1}$ still equals $d_{0}$ (see [Baker and Rumely 2010, §2.3, §9; Benedetto 2010, §6.3]): in particular, the extended action of $\phi$ on $\mathrm{P}^{1}$ induces a push-forward $\phi_{*}$ and a pullback $\phi^{*}$ on the spaces of continuous functions and of Radon measures on $\mathrm{P}^{1}$. When $d_{0}=0$, the extended $\phi$ is still constant, and we set $\phi^{*} \mu:=0$ on $\mathrm{P}^{1}$ for every Radon measure $\mu$ on $\mathrm{P}^{1}$ by convention. Let $F_{\phi} \in K\left[p_{0}, p_{1}\right]_{\operatorname{deg} \phi} \times K\left[p_{0}, p_{1}\right]_{\operatorname{deg} \phi}$ be a lift of $\phi$. The function 


$$
T_{F_{\phi}}:=\log \left\|F_{\phi}(\cdot /\|\cdot\|)\right\|=\log \left\|F_{\phi}\right\|-(\operatorname{deg} \phi) \log \|\cdot\|
$$

on $K^{2} \backslash\{0\}$ descends to $\mathbb{P}^{1}$ and in turn extends continuously to $\mathrm{P}^{1}$, satisfying $\Delta T_{F_{\phi}}=\phi^{*} \Omega_{\text {can }}-(\operatorname{deg} \phi) \Omega_{\text {can }}$ on $\mathrm{P}^{1}$ (see, e.g., [Okuyama 2013a, Definition 2.8]). Moreover, $\phi$ is a Lipschitz continuous endomorphism on $\left(\mathrm{P}^{1}, \mathrm{~d}\right)$ and $T_{F_{\phi}}$ is a Lipschitz continuous function on $\left(\mathrm{P}^{1}, \mathrm{~d}\right)$ (for nonarchimedean $K$, see [Baker and Rumely 2010, Proposition 9.37]). For every $n \in \mathbb{N}$, the homogeneous polynomial $F_{\phi}^{n} \in K\left[p_{0}, p_{1}\right]_{\operatorname{deg} \phi^{n}} \times K\left[p_{0}, p_{1}\right]_{\operatorname{deg} \phi^{n}}$ is a lift of $\phi^{n}$.

Let $f \in K(z)$ be a rational function of degree $d>1$, and consider a lift $F \in K\left[p_{0}, p_{1}\right]_{d} \times K\left[p_{0}, p_{1}\right]_{d}$ of $f$. The uniform limit $g_{F}:=\lim _{n \rightarrow \infty} T_{F^{n}} / d^{n}$ on $\mathrm{P}^{1}$ exists, and more precisely, for every $n \in \mathbb{N}$,

$$
\sup _{\mathrm{P}^{1}}\left|g_{F}-\frac{T_{F^{n}}}{d^{n}}\right| \leq \frac{\sup _{\mathrm{P}^{1}}\left|T_{F}\right|}{d^{n}(d-1)}
$$

The limit $g_{F}$ is called the dynamical Green function of $F$ on $\mathrm{P}^{1}$ and is a continuous weight on $\mathrm{P}^{1}$. The probability Radon measure

$$
\mu_{f}:=\mu^{g_{F}}=\Delta g_{F}+\Omega_{\text {can }}=\lim _{n \rightarrow \infty} \frac{\left(f^{n}\right)^{*} \Omega_{\text {can }}}{d^{n}} \quad \text { weakly on } \mathrm{P}^{1}
$$

is independent of the choice of $F$ and satisfies $f^{*} \mu_{f}=d \cdot \mu_{f}$ on $\mathrm{P}^{1}$. It is called the $f$-equilibrium (or canonical) measure on $\mathrm{P}^{1}$. Moreover, $g_{F}$ is a Hölder continuous function on $\left(\mathrm{P}^{1}, \mathrm{~d}\right)$ (for nonarchimedean $K$, see [Favre and Rivera-Letelier 2006, §6.6]). The remarkable energy formula

$$
V_{g_{F}}=-\frac{\log |\operatorname{Res} F|}{d(d-1)}
$$

was first established by DeMarco [2003] for archimedean $K$ and was generalized to rational functions defined over a number field by Baker and Rumely [2006] (for a simple proof of (8-3) which also works for general $K$, see [Baker 2009, Appendix A] or [Okuyama and Stawiska 2011, Appendix]). The dynamical Green function $g_{f}$ of $f$ on $\mathrm{P}^{1}$ is the unique normalized weight on $\mathrm{P}^{1}$ such that $\mu^{g_{f}}=\mu_{f}$, i.e., for any lift $F$ of $f, g_{f} \equiv g_{F}+V_{g_{F}} / 2$ on $\mathrm{P}^{1}$.

8.2. A Berkovich space version of the quasiperiodicity region $\mathcal{E}_{f}$. For nonarchimedean dynamics, see [Baker and Rumely 2010, §10; Favre and Rivera-Letelier 2010, §2.3; Benedetto 2010, §6.4]. For complex dynamics, see, e.g., [Milnor 2006].

Let $f \in K(z)$ be a rational function of degree $>1$. The Berkovich Julia set of $f$ is

$$
J(f):=\left\{\mathcal{S} \in \mathrm{P}^{1}: \bigcap_{U \text { open in } \mathrm{P}^{1} \text { containing } \mathcal{S}}\left(\bigcup_{n \in \mathbb{N}} f^{n}(U)\right)=\mathrm{P}^{1} \backslash E(f)\right\},
$$

where $E(f):=\left\{a \in \mathbb{P}^{1}: \# \bigcup_{n \in \mathbb{N}} f^{-n}(a)<\infty\right\}$ is the exceptional set of $f$. The 
Berkovich Fatou set is $\mathrm{F}(f):=\mathrm{P}^{1} \backslash \mathrm{J}(f)$. By definition, $\mathrm{J}(f)$ is closed and $\mathrm{F}(f)$ is open in $\mathrm{P}^{1}$, both $\mathrm{J}(f)$ and $\mathrm{F}(f)$ are totally invariant under $f$, and $\mathrm{J}(f)$ has no interior point unless $\mathrm{J}(f)=\mathrm{P}^{1}$. The classical Julia set $\mathrm{J}(f) \cap \mathbb{P}^{1}$ (resp. the classical Fatou set $\left.\mathrm{F}(f) \cap \mathbb{P}^{1}\right)$ coincides with the set of all nonequicontinuity points (resp. the region of local equicontinuity) of the family $\left\{f^{n}: n \in \mathbb{N}\right\}$ as a family of endomorphisms on $\left(\mathbb{P}^{1},[z, w]\right)$.

A component $U$ of $\mathrm{F}(f)$ is called a Berkovich Fatou component of $f$, and is said to be cyclic under $f$ if $f^{n}(U)=U$ for some $n \in \mathbb{N}$, which is called a period of $U$ under $f$. Following [Fatou 1920, §28], a cyclic Berkovich Fatou component $U$ of $f$ having a period $n \in \mathbb{N}$ is called a singular domain of $f$ if $f^{n}: U \rightarrow U$ is injective. Let $\mathcal{E}_{f}$ be the set of all points $\mathcal{S} \in \mathrm{P}^{1}$ having an open neighborhood $V$ in $\mathrm{P}^{1}$ such that $\liminf _{n \rightarrow \infty} \sup _{V \cap \mathbb{P}^{1}}\left[f^{n}, \mathrm{Id}\right]=0$, which is a Berkovich space version of RiveraLetelier's quasiperiodicity region of $f$. When $K$ is archimedean, $\mathcal{E}_{f}$ coincides with the union of all singular domains of $f$, and when $K$ is nonarchimedean, $\mathcal{E}_{f}$ is still open and forward invariant under $f$ and is contained in the union of all singular domains of $f$ (see [Okuyama 2013a, Lemma 4.4]).

The following function $T_{*}$ is Rivera-Letelier's iterative logarithm of $f$ on $\mathcal{E}_{f} \cap \mathbb{P}^{1}$, which is a nonarchimedean counterpart of the uniformization of a Siegel disk or a Herman ring of $f$.

Theorem 8.3 ([Rivera-Letelier 2003, §3.2, §4.2]. See also [Favre and Rivera-Letelier 2010, Théorème 2.15]). Suppose that $K$ is nonarchimedean and has characteristic 0 and residual characteristic $p$. Let $f \in K(z)$ be a rational function on $\mathbb{P}^{1}$ of degree $>1$ and suppose that $\mathcal{E}_{f} \neq \varnothing$, which implies $p>0$ by [Favre and RiveraLetelier 2010, Lemme 2.14]. Then for every component $Y$ of $\mathcal{E}_{f}$ not containing $\infty$, there are $k_{0} \in \mathbb{N}$, a continuous action $T: \mathbb{Z}_{p} \times(Y \cap K) \ni(\omega, y) \mapsto T^{\omega}(y) \in Y \cap K$, and a nonconstant $K$-valued holomorphic function $T_{*}$ on $Y \cap K$ such that for every $m \in \mathbb{Z},\left(f^{k_{0}}\right)^{m}=T^{m}$ on $Y \cap K$, that for every $\omega \in \mathbb{Z}_{p}, T^{\omega}$ is a biholomorphism on $Y \cap K$, and that for every $\omega_{0} \in \mathbb{Z}_{p}$,

$$
\lim _{\mathbb{Z}_{p} \ni \omega \rightarrow \omega_{0}} \frac{T^{\omega}-T^{\omega_{0}}}{\omega-\omega_{0}}=T_{*} \circ T^{\omega_{0}} \quad \text { locally uniformly on } Y \cap K \text {. }
$$

8.3. The fundamental relationship between $\mu_{f}$ and $\mathrm{J}(f)$. If $K$ is archimedean, the inclusion supp $\mu_{f} \subset \mathrm{J}(f)$ is classical, but it is not trivial from the definition of $\mathrm{J}(f)$ when $K$ is nonarchimedean. For an elementary proof, see [Okuyama 2013a, proof of Theorem 2.18]. Actually the equality supp $\mu_{f}=\mathrm{J}(f)$ holds, but we will dispense with the reverse (and easier) inclusion $\mathrm{J}(f) \subset \operatorname{supp} \mu_{f}$.

\section{Proofs of Theorems 3 and 4}

Let $k$ be a product formula field. The proof of the following is based not only on (PF) but also on elimination theory (and the strong triangle inequality). 
Theorem 9.1 [Baker and Rumely 2006, Lemma 3.1]. Let $k$ be a product formula field. For every $\phi \in k(z)$ and every lift $F_{\phi} \in k\left[p_{0}, p_{1}\right]_{\operatorname{deg} \phi} \times k\left[p_{0}, p_{1}\right]_{\operatorname{deg} \phi}$ of $\phi$, there exists a finite subset $E_{F_{\phi}}$ in $M_{k}$ containing all the infinite places of $k$ such that for every $v \in M_{k} \backslash E_{F_{\phi}}$, we have $\left|\operatorname{Res} F_{\phi}\right|_{v}=1$ and $\left\|F_{\phi}(\cdot)\right\|_{v}=\|\cdot\|_{v}^{\operatorname{deg} \phi}$ on $\mathbb{C}_{v}^{2}$.

Let $f \in k(z)$ be a rational function of degree $>1$ and $F \in k\left[p_{0}, p_{1}\right]_{d} \times k\left[p_{0}, p_{1}\right]_{d}$ a lift of $f$. Then the family $\hat{g}_{f}=\left\{g_{f, v}: v \in M_{k}\right\}$ is an adelic normalized weight, where $g_{f, v}$ is the dynamical Green function of $f$ on $\mathrm{P}^{1}\left(\mathbb{C}_{v}\right)$ for every $v \in M_{k}$. Indeed, letting $g_{F, v}$ be the dynamical Green function of $F$ on $\mathrm{P}^{1}\left(\mathbb{C}_{v}\right)$ for each $v \in M_{k}$ and $E_{F}$ be a finite subset in $M_{k}$ obtained by Theorem 9.1 applied to $F$, for every $v \in M_{k} \backslash E_{F}$ we have $T_{F^{n}, v} \equiv 0$ on $\mathrm{P}^{1}\left(\mathbb{C}_{v}\right)$ for every $n \in \mathbb{N}$, giving $g_{f, v} \equiv g_{F, v} \equiv 0$ on $\mathrm{P}^{1}\left(\mathbb{C}_{v}\right)$. We call the adelic normalized weight $\hat{g}_{f}=\left\{g_{f, v}: v \in M_{k}\right\}$ and the adelic probability measure $\hat{\mu}_{f}:=\mu^{\hat{g}_{f}}$ the adelic dynamical Green function of $f$ and the adelic $f$ equilibrium (or canonical) measure, respectively. Here, for every $v \in M_{k}, \mu_{f, v}:=$ $\mu^{g_{f, v}}=\mu_{v}^{\hat{g}_{f}}$ (as in Section 1) is the $f$-equilibrium (or canonical) measure on $\mathrm{P}^{1}\left(\mathbb{C}_{v}\right)$.

Lemma 9.2. Let $k$ be a product formula field. Let $f, a \in k(z)$ be rational functions and suppose $d:=\operatorname{deg} f>1$. Then the sequence $\left(\left[f^{n}=a\right]\right)$ of $k$-effective divisors on $\mathbb{P}^{1}(\bar{k})$ has strictly small $\hat{g}_{f}$-heights in that

$$
\limsup _{n \rightarrow \infty}\left(d^{n}+\operatorname{deg} a\right) \cdot h_{\hat{g}_{f}}\left(\left[f^{n}=a\right]\right)<\infty .
$$

Proof. Let $F \in k\left[p_{0}, p_{1}\right]_{d} \times k\left[p_{0}, p_{1}\right]_{d}$ and $A \in k\left[p_{0}, p_{1}\right]_{\operatorname{deg} a} \times k\left[p_{0}, p_{1}\right]_{\operatorname{deg} a}$ be lifts of $f$ and $a$, respectively. Then $F^{n} \wedge A \in k\left[p_{0}, p_{1}\right]_{d^{n}+\operatorname{deg} a} \times k\left[p_{0}, p_{1}\right]_{d^{n}+\operatorname{deg} a}$ is a representative of $\left[f^{n}=a\right]$ for every $n \in \mathbb{N}$ such that $f^{n} \not \equiv a$. Let $E_{F}, E_{A}$ be finite subsets in $M_{k}$ obtained by applying Theorem 9.1 to $F, A$, respectively, so that for every $v \in M_{k} \backslash\left(E_{F} \cup E_{A}\right)$ and every $n \in \mathbb{N}$, we have $T_{F^{n}, v} \equiv T_{A, v} \equiv 0$ and $g_{F, v} \equiv 0$ on $\mathrm{P}^{1}\left(\mathbb{C}_{v}\right)$. For every $v \in M_{k}$ and every sufficiently large $n \in \mathbb{N}$, since $\left|F^{n} \wedge A\right|_{v} \leq\left\|F^{n}\right\|_{v}\|A\|_{v}$ on $\mathbb{C}_{v}^{2} \backslash\{0\}$, we have $\log S_{F^{n} \wedge A, v} \leq T_{F^{n}, v}+T_{A, v}$ on $\mathbb{P}^{1}\left(\mathbb{C}_{v}\right)$ and in turn on $\mathrm{P}^{1}\left(\mathbb{C}_{v}\right)$ (recalling that $S_{F^{n} \wedge A, v}=\left|\left(F^{n} \wedge A\right)\left(\cdot /\|\cdot\|_{v}\right)\right|_{v}$ on $\left.\mathbb{P}^{1}\left(\mathbb{C}_{v}\right)\right)$, so using also $g_{f, v} \equiv g_{F, v}+V_{g_{F, v}} / 2$ on $\mathrm{P}^{1}\left(\mathbb{C}_{v}\right)$, we obtain

$$
\frac{\log S_{F^{n} \wedge A, v}}{d^{n}+\operatorname{deg} a}-g_{f, v} \leq \frac{T_{F^{n}, v}+T_{A, v}}{d^{n}+\operatorname{deg} a}-\left(g_{F, v}+\frac{1}{2} V_{g_{F, v}}\right) \quad \text { on } \mathrm{P}^{1}\left(\mathbb{C}_{v}\right) .
$$

Hence, by the definition (1-1) of $h_{\hat{g}_{f}}$, the Jensen-type formula (2-11), the energy formula (8-3) (with Res $F \in k \backslash\{0\}$ ), and (PF), we have

$$
\begin{aligned}
h_{\hat{g}_{f}}\left(\left[f^{n}=a\right]\right) & \leq \sum_{v \in M_{k}} N_{v} \int_{\mathrm{P}^{1}\left(\mathbb{C}_{v}\right)}\left(\frac{T_{F^{n}, v}+T_{A, v}}{d^{n}+\operatorname{deg} a}-g_{F, v}\right) \mathrm{d} \mu_{f, v}-\frac{3}{2} \sum_{v \in M_{k}} N_{v} \cdot V_{g_{F, v}} \\
& =\sum_{v \in E_{F} \cup E_{A}} N_{v} \int_{\mathrm{P}^{1}\left(\mathbb{C}_{v}\right)}\left(\frac{T_{F^{n}, v}+T_{A, v}}{d^{n}+\operatorname{deg} a}-g_{F, v}\right) \mathrm{d} \mu_{f, v} \\
& =O\left(d^{-n}\right) \quad \text { as } n \rightarrow \infty,
\end{aligned}
$$


where the final order estimate is by $(8-2)$ and $\#\left(E_{F} \cup E_{A}\right)<\infty$.

With the help of Lemma 9.2, Theorems 3 and 4 follow from Theorems 1 and 2, respectively.

We omit the proof of the following characterization of $h_{\hat{g}_{f}}$, which we will dispense with in this article.

Lemma 9.3. Let $k$ be a product formula field. Then for every rational function $f \in k(z)$ of degree $d>1$, the $\hat{g}_{f}$-height function $h_{\hat{g}_{f}}$ coincides with the CallSilverman $f$-dynamical (or canonical) height function in that for every $k$-effective divisor $\mathcal{Z}$ on $\mathbb{P}^{1}(\bar{k}),\left(f_{*} \mathcal{Z}\right.$ is also a $k$-effective divisor on $\mathbb{P}^{1}(\bar{k})$, and $)$ the equality $\left(h_{\hat{g}_{f}} \circ f_{*}\right)(\mathcal{Z})=\left(d \cdot h_{\hat{g}_{f}}\right)(\mathcal{Z})$ holds.

\section{Proofs of Theorems 5 and 6}

Let $K$ be an algebraically closed field that is complete with respect to a nontrivial absolute value $|\cdot|$. For subsets $A, B \subset \mathbb{P}^{1}$, set $[A, B]:=\inf _{z \in A, z^{\prime} \in B}\left[z, z^{\prime}\right]$.

Let $f, a \in K(z)$ be rational functions and suppose that $d:=\operatorname{deg} f>1$. Let $N \in \mathbb{N}$ be so large that $f^{n} \not \equiv a$ if $n>N$. Then $\left(\overline{\bigcup_{n>N} \operatorname{supp}\left[f^{n}=a\right]} \cup \mathrm{J}(f)\right) \cap \mathbb{P}^{1}$ is closed in $\mathbb{P}^{1}$.

Lemma 10.1. Suppose that $K$ has characteristic 0 . Let $D$ be a chordal disk in $\mathbb{P}^{1}$ of radius $>0$ satisfying $\liminf \operatorname{in}_{n \rightarrow \infty} \sup _{D}\left[f^{n}, a\right]=0$. Then:

(i) $a(D) \subset \mathcal{E}_{f}$.

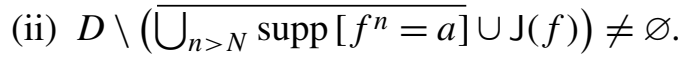

(iii) There is a chordal disk $D^{\prime}$ in $\mathbb{P}^{1} \backslash \mathrm{J}(f)$ of radius $>0$ such that

$$
\liminf _{n \rightarrow \infty}\left[f^{n}\left(D^{\prime}\right), a\left(D^{\prime}\right)\right]>0 .
$$

Proof of (i). Since $\liminf _{n \rightarrow \infty} \sup _{D}\left[f^{n}, a\right]=0$, there is a sequence $\left(n_{j}\right)$ in $\mathbb{N}$ such that $\lim _{j \rightarrow \infty} \sup _{D}\left[f^{n_{j}}, a\right]=0$ and $\lim _{j \rightarrow \infty}\left(n_{j+1}-n_{j}\right)=\infty$. For every $z \in D$, set $D^{\prime \prime}:=\left\{w \in \mathbb{P}^{1}:[w, a(z)] \leq r\right\}$ in $a(D)$ for $r>0$ small enough. Then $\liminf _{j \rightarrow \infty} \sup _{D^{\prime \prime}}\left[f^{n_{j+1}-n_{j}}, \mathrm{Id}\right] \leq \lim \sup _{j \rightarrow \infty} \sup _{D}\left[f^{n_{j+1}}, f^{n_{j}}\right]=0$, so that $a(z) \in \mathcal{E}_{f}$. Hence $a(D) \subset \mathcal{E}_{f}$.

Proof of (ii). When $K$ is archimedean, let $Y$ be the component of $\mathcal{E}_{f}$ containing $a(D)$, which is by the first assertion either a Siegel disk or a Herman ring of $f$. Setting $k_{0}:=\min \left\{n \in \mathbb{N}: f^{n}(Y)=Y\right\}$, there are a sequence $\left(n_{j}\right)$ and an $N$ in $\mathbb{N}$ with the properties that $f^{n_{N}}(D) \subset Y$, that $k_{0} \mid\left(n_{j}-n_{N}\right)$ for every $j \geq N$, and that $a=\lim _{j \rightarrow \infty}\left(f^{k_{0}}\right)^{\left(n_{j}-n_{N}\right) / k_{0}} \circ f^{n_{N}}$ uniformly on $D$. Then $D \cap \mathrm{J}(f)=\varnothing$. Let $\lambda \in \mathbb{C}$ be the rotation number of $Y$, so that there exists a holomorphic injection $h: Y \rightarrow \mathbb{C}$ such that $h \circ f^{k_{0}}=\lambda \cdot h$ on $Y$. Then $|\lambda|=1$ but $\lambda$ is not a root of unity (by $d>1)$. Choosing a subsequence of $\left(n_{j}\right)$ if necessary, $\lambda_{a}:=\lim _{j \rightarrow \infty} \lambda^{\left(n_{j}-n_{N}\right) / k_{0}} \in \mathbb{C}$ 
exists. For every $n \geq n_{N}$, if $k_{0} \nmid\left(n-n_{N}\right)$, then $D \cap \operatorname{supp}\left[f^{n}=a\right]=\varnothing$, whereas if $k_{0} \mid\left(n-n_{N}\right)$, then $h \circ f^{n}-h \circ a=\left(\lambda^{\left(n-n_{N}\right) / k_{0}}-\lambda_{a}\right) \cdot\left(h \circ f^{n_{N}}\right)$ on $D$, so $\left(D \backslash\left(h \circ f^{n_{N}}\right)^{-1}(0)\right) \cap \operatorname{supp}\left[f^{n}=a\right]=\varnothing$ if $n$ is large enough.

When $K$ is nonarchimedean, let $Y$ be the component of $\mathcal{E}_{f}$ containing $a(D)$. Without loss of generality, we assume that $\infty \notin Y$, and then applying Theorem 8.3 to this $Y$, we obtain $p \in \mathbb{N}, k_{0} \in \mathbb{N}, T$, and $T_{*}$ as in the theorem. There are a sequence $\left(n_{j}\right)$ and an $N$ in $\mathbb{N}$ such that $f^{n_{N}}(D) \subset Y, k_{0} \mid\left(n_{j}-n_{N}\right)$ for every $j \geq N$, and $a=\lim _{j \rightarrow \infty}\left(f^{k_{0}}\right)^{\left(n_{j}-n_{N}\right) / k_{0}} \circ f^{n_{N}}$ uniformly on $D$. Then $D \cap \mathrm{J}(f)=\varnothing$. Choosing a subsequence of $\left(n_{j}\right)$ if necessary, $\omega_{a}:=\lim _{j \rightarrow \infty}\left(n_{j}-n_{N}\right) / k_{0} \in \mathbb{Z}_{p}$ exists. For every $n \geq n_{N}$, if $k_{0} \nmid\left(n-n_{N}\right)$, then $D \cap \operatorname{supp}\left[f^{n}=a\right]=\varnothing$, whereas if $k_{0} \mid\left(n-n_{N}\right)$, then

$$
f^{n}-a=\left(T^{\left(n-n_{N}\right) / k_{0}}-T^{\omega_{a}}\right) \circ f^{n_{N}}
$$

on $D$. Choose $b \in D \backslash\{\infty\}$ and $r \in\left|K^{*}\right|$ small enough that the (K-closed) disk $B=\{z \in K:|z-b| \leq r\}$ is contained in $D$, and fix $\epsilon \in\left|K^{*}\right|$ so small that for $Z_{\epsilon}:=\bigcup_{w \in B \cap\left(T_{*} \circ T^{\omega_{a}} \circ f^{n_{N}}\right)^{-1}(0)}\{z \in B:|z-w|<\epsilon\}$, we have $B \backslash Z_{\epsilon} \neq \varnothing$. The maximum modulus principle from rigid analysis (see [Bosch, Güntzer, and Remmert

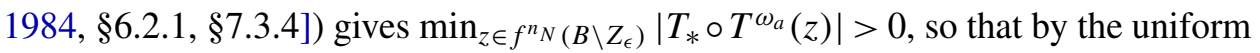
convergence (8-4) and the equality (10-1), $\left(B \backslash Z_{\epsilon}\right) \cap \operatorname{supp}\left[f^{n}=a\right]=\varnothing$ if $n$ is large enough.

Proof of (iii). By the first assertion, there is a unique singular domain $U$ of $f$ containing $a(D)$. Fix $n_{0} \in \mathbb{N}$ such that $f^{n_{0}}(U)=U$, and set $\mathcal{C}:=\bigcup_{j=0}^{n_{0}-1} f^{j}(U)$. Then there is a component $V$ of $f^{-1}(\mathcal{C}) \backslash \mathcal{C}$ since $f: \mathcal{C} \rightarrow \mathcal{C}$ is injective and $d>1$. Fix a chordal disk $D^{\prime \prime}$ of radius $>0$ in $a^{-1}(V) \cap\left(\mathbb{P}^{1} \backslash J(f)\right)$, so that $a\left(D^{\prime \prime}\right) \subset V \subset f^{-1}(\mathcal{C}) \backslash \mathcal{C}$. If $a\left(D^{\prime \prime}\right) \cap \bigcup_{n \in \mathbb{N} \cup\{0\}} f^{n}\left(D^{\prime \prime}\right)=\varnothing$, then we are done by setting $D^{\prime}=\left\{z \in \mathbb{P}^{1}:[z, b] \leq r\right\}$ for some $b \in D^{\prime \prime}$ and $r>0$ small enough. But if there is $N \in \mathbb{N} \cup\{0\}$ such that $a\left(D^{\prime \prime}\right) \cap f^{N}\left(D^{\prime \prime}\right) \neq \varnothing$, then by setting $D^{\prime}:=\left\{z \in \mathbb{P}^{1}:[z, b] \leq r\right\}$ for some $b \in D^{\prime \prime} \cap f^{-N}\left(a\left(D^{\prime \prime}\right)\right)$ and $r>0$ small enough, we get $\liminf _{n \rightarrow \infty}\left[a\left(D^{\prime}\right), f^{n}\left(D^{\prime}\right)\right]>0$ from

$$
a\left(D^{\prime}\right) \cap \bigcup_{n \geq N+1} f^{n}\left(D^{\prime}\right) \subset a\left(D^{\prime \prime}\right) \cap \bigcup_{n \in \mathbb{N}} f^{n}\left(a\left(D^{\prime \prime}\right)\right) \subset V \cap \mathcal{C}=\varnothing .
$$

Lemma 10.2. For every $w_{0} \in \mathbb{P}^{1} \backslash\left(\bigcup_{n>N} \operatorname{supp}\left[f^{n}=a\right] \cup J(f)\right)$, there is a function $\phi_{0} \in C^{1}\left(\mathrm{P}^{1}\right)$ such that $\phi_{0} \equiv \log \left[w_{0}, \cdot\right]_{\text {can }}$ on $\bigcup_{n>N} \operatorname{supp}\left[f^{n}=a\right] \cup \mathrm{J}(f)$.

Proof. Fix $w_{0} \in \mathbb{P}^{1} \backslash\left(\overline{\bigcup_{n>N} \operatorname{supp}\left[f^{n}=a\right]} \cup \mathrm{J}(f)\right)$. Without loss of generality, we can assume that $w_{0} \neq \infty$, and fix $\epsilon>0$ so small that

$$
\left\{\mathcal{S} \in \mathrm{P}^{1}:\left|\mathcal{S}-w_{0}\right|_{\infty} \leq \epsilon\right\} \subset \mathrm{P}^{1} \backslash\left(\bigcup_{n>N} \operatorname{supp}\left[f^{n}=a\right] \cup \mathrm{J}(f)\right)
$$

(recall Sections 3.1 and 3.2 here). 
When $K$ is nonarchimedean, by the definition of the map $\pi_{\epsilon}: \mathrm{A}^{1} \rightarrow \mathrm{A}^{1}$, we have $\left\{\mathcal{S} \in \mathrm{P}^{1}: \mathcal{S} \preceq \pi_{\epsilon}\left(w_{0}\right)\right\}=\left\{\mathcal{S} \in \mathrm{P}^{1}:\left|\mathcal{S}-w_{0}\right|_{\infty} \leq \epsilon\right\}$. The function

$$
\mathcal{S} \mapsto \phi_{0}(\mathcal{S}):=\left\{\begin{array}{lll}
\log \left[w_{0}, \pi_{\epsilon}\left(w_{0}\right)\right]_{\text {can }} & \text { if } \mathcal{S} \preceq \pi_{\epsilon}\left(w_{0}\right), & \text { on } \mathrm{P}^{1} \\
\log \left[w_{0}, \mathcal{S}\right]_{\text {can }} & \text { otherwise }
\end{array}\right.
$$

is in $C^{1}\left(\mathrm{P}^{1}\right)$ since it is continuous on $\mathrm{P}^{1}$, locally constant on $\mathrm{P}^{1}$ except for the segment $\mathcal{I}$ in $\mathrm{H}^{1}$ joining $\pi_{\epsilon}\left(w_{0}\right)$ and $\mathcal{S}_{\text {can }}$, and linear on $\mathcal{I}$ with respect to the length parameter induced by the hyperbolic metric $\rho$ on $\mathrm{H}^{1}$. When $K$ is archimedean (so $\left.\mathrm{P}^{1} \cong \mathbb{P}^{1}\right)$, there is a function $\phi_{0} \in C^{1}\left(\mathbb{P}^{1}\right)$ satisfying

$$
z \mapsto \phi_{0}(z)= \begin{cases}\int_{\mathbb{P} 1} \log \left[w_{0}, w\right] \mathrm{d}[z]_{\epsilon / 2}(w) & \text { if }\left|z-w_{0}\right| \leq \epsilon / 2, \\ \log \left[w_{0}, z\right] & \text { if }\left|z-w_{0}\right| \geq \epsilon \text { or } z=\infty .\end{cases}
$$

In both cases, the given $\phi_{0} \in C^{1}\left(\mathrm{P}^{1}\right)$ satisfies the desired property.

Fact 10.3. For rational functions $\phi, \psi \in K(z)$, the chordal proximity function

$$
\mathcal{S} \mapsto[\phi, \psi]_{\text {can }}(\mathcal{S}) \quad \text { on } \mathrm{P}^{1}
$$

between $\phi$ and $\psi$ is the unique continuous extension of the function $z \mapsto[\phi(z), \psi(z)]$ on $\mathbb{P}^{1}$ to $\mathrm{P}^{1}$ (see [Okuyama 2013a, Proposition 2.9] for its construction, as well as Remark 2.10 of the same paper), and for every continuous weight $g$ on $\mathrm{P}^{1}$, we also define its weighted version by $\Phi(\phi, \psi)_{g}:=\log [\phi, \psi]_{\text {can }}-g \circ \phi-g \circ \psi$ on $\mathrm{P}^{1}$.

For every $n \in \mathbb{N}$ such that $f^{n} \not \equiv a$, recall the Riesz decomposition

$$
\Phi\left(f^{n}, a\right)_{g_{f}}=U_{g_{f},\left[f^{n}=a\right]-\left(d^{n}+\operatorname{deg} a\right) \mu_{f}}-U_{g_{f}, a^{*} \mu_{f}}+\int_{\mathrm{P}^{1}} \Phi\left(f^{n}, a\right)_{g_{f}} \mathrm{~d} \mu_{f}
$$

on $\mathrm{P}^{1}$, and also $U_{g_{f}, a^{*} \mu_{f}}=g_{f} \circ a+U_{g_{f}, a^{*} \Omega_{\mathrm{can}}}-\int_{\mathrm{P}^{1}}\left(g_{f} \circ a\right) \mathrm{d} \mu_{f}$ on $\mathrm{P}^{1}$ [Okuyama 2013a, Lemma 2.19].

Proof of Theorem 5. Let $k$ be a product formula field of characteristic 0 . Let $f \in k(z)$ be a rational function of degree $d>1$ and $a \in k(z)$ a rational function of degree $>0$. Let $N \in \mathbb{N}$ be so large that $f^{n} \not \equiv a$ if $n>N$. Fix $v \in M_{k}$. Let $D$ be a chordal disk in $\mathbb{P}^{1}\left(\mathbb{C}_{v}\right)$ of radius $>0$, and assume that $\liminf _{n \rightarrow \infty} \sup _{D}\left[f^{n}, a\right]_{v}=0$; otherwise we are done. By Lemma 10.1, there are not only a point $w_{0} \in D \backslash$ $\left(\overline{\bigcup_{n>N}\left[f^{n}=a\right]} \cup \mathrm{J}(f)_{v}\right)$ but also a chordal disk $D^{\prime}$ in $\mathbb{P}^{1}\left(\mathbb{C}_{v}\right) \backslash \mathrm{J}(f)_{v}$ of radius $>0$ such that $\liminf _{n \rightarrow \infty}\left[f^{n}\left(D^{\prime}\right), a\left(D^{\prime}\right)\right]_{v}>0$. Fix a point $w_{1} \in D^{\prime}$. Then also $w_{1} \in \mathbb{P}^{1} \backslash\left(\overline{\bigcup_{n>N}\left[f^{n}=a\right]} \cup \mathrm{J}(f)_{v}\right)$.

For every $n \in \mathbb{N}$ large enough and every $j \in\{0,1\}$, by (10-2),

$(10-3) \quad \log \left[f^{n}\left(w_{j}\right), a\left(w_{j}\right)\right]_{v}-g_{f, v}\left(f^{n}\left(w_{j}\right)\right)-g_{f, v}\left(a\left(w_{j}\right)\right)$

$$
=U_{g_{f, v},\left[f^{n}=a\right]-\left(d^{n}+\operatorname{deg} a\right) \mu_{f, v}}\left(w_{j}\right)-U_{g_{f, v}, a^{*} \mu_{f, v}}\left(w_{j}\right)+\int_{\mathrm{P}^{1}\left(\mathbb{C}_{v}\right)} \Phi\left(f^{n}, a\right)_{g_{f, v}} \mathrm{~d} \mu_{f, v},
$$


so that taking the difference of both sides in (10-3) for each $j \in\{0,1\}$ and noting that $g_{f, v}$ and $U_{g_{f, v}, a^{*} \mu_{f, v}}$ are bounded on $\mathrm{P}^{1}\left(\mathbb{C}_{v}\right)$, we have

$$
\begin{aligned}
\log \left[f^{n}\left(w_{0}\right),\right. & \left.a\left(w_{0}\right)\right]_{v}-\log \left[f^{n}\left(w_{1}\right), a\left(w_{1}\right)\right]_{v} \\
= & \int_{\mathrm{P}^{1}\left(\mathbb{C}_{v}\right)} \log \left[w_{0}, \mathcal{S}^{\prime}\right]_{\mathrm{can}, v} \mathrm{~d}\left(\left[f^{n}=a\right]-\left(d^{n}+\operatorname{deg} a\right) \mu_{f}\right)\left(\mathcal{S}^{\prime}\right) \\
& \quad-\int_{\mathrm{P}^{1}\left(\mathbb{C}_{v}\right)} \log \left[w_{1}, \mathcal{S}^{\prime}\right]_{\operatorname{can}, v} \mathrm{~d}\left(\left[f^{n}=a\right]-\left(d^{n}+\operatorname{deg} a\right) \mu_{f}\right)\left(\mathcal{S}^{\prime}\right)+O(1)
\end{aligned}
$$

as $n \rightarrow \infty$. In the left hand side, by the choice of $w_{0}$ and $w_{1}$, we have

$$
\log \sup _{D}\left[f^{n}, a\right]_{v} \geq \log \left[f^{n}\left(w_{0}\right), a\left(w_{0}\right)\right]_{v}
$$

and

$$
\liminf _{n \rightarrow \infty} \log \left[f^{n}\left(w_{1}\right), a\left(w_{1}\right)\right]_{v} \geq \liminf _{n \rightarrow \infty} \log \left[f^{n}\left(D^{\prime}\right), a\left(D^{\prime}\right)\right]_{v}>-\infty,
$$

so that as $n \rightarrow \infty$,

$$
\log \sup _{D}\left[f^{n}, a\right]_{v}+O(1) \geq \log \left[f^{n}\left(w_{0}\right), a\left(w_{0}\right)\right]_{v}-\log \left[f^{n}\left(w_{1}\right), a\left(w_{1}\right)\right]_{v} .
$$

In the right hand side, for each $j \in\{0,1\}$, by Lemma 10.2 applied to $w_{j}$, the inclusion supp $\mu_{f} \subset \mathrm{J}(f)$, and Theorem 3 (and $k_{s}=\bar{k}$ in the characteristic 0 case), we have

$$
\begin{aligned}
\int_{\mathrm{P}^{1}\left(\mathbb{C}_{v}\right)} \log \left[w_{j}, \mathcal{S}^{\prime}\right]_{\mathrm{can}, v} \mathrm{~d}\left(\left[f^{n}=a\right]-\left(d^{n}+\operatorname{deg} a\right) \mu_{f}\right)\left(\mathcal{S}^{\prime}\right) \\
=O\left(\sqrt{n \cdot\left(\left[f^{n}=a\right] \times\left[f^{n}=a\right]\right)\left(\operatorname{diag}_{\mathbb{P}^{1}(\bar{k})}\right)}\right) \quad \text { as } n \rightarrow \infty .
\end{aligned}
$$

These estimates complete the proof of (1-4) for this $v \in M_{k}$.

Fact 10.4. For a rational function $f(z) \in k(z)$ over a field $k$, a point $w \in \mathbb{P}^{1}(\bar{k})$ is called a multiple periodic point of $f$ if $\left[f^{n}=\operatorname{Id}\right](\{w\})>1$ for some $n \in \mathbb{N}$. For a rational function $f(z) \in k(z)$ over a field $k$ of characteristic 0 , there are at most finitely many multiple periodic points of $f$ in $\mathbb{P}^{1}(\bar{k})$; this is well known in the case that $k=\mathbb{C}$ (see, e.g., [Milnor 2006, §13]), and holds in general by the Lefschetz principle (see, e.g., [Eklof 1973]).

Proof of Theorem 6. As noted above, $f$ has at most finitely many multiple periodic points in $\mathbb{P}^{1}(\bar{k})$, and for every multiple periodic point $w$ of $f$, setting $p=p_{w}:=\min \left\{n \in \mathbb{N}:\left[f^{n}=\operatorname{Id}\right](\{w\})>1\right\}$, by the (formal) power series expansion $f^{p}(z)=w+(z-w)+C(z-w)^{\left[f^{p}=\mathrm{Id}\right](\{w\})}+\cdots$ of $f^{p}$ around $w$, we also have $\sup _{n \in \mathbb{N}}\left[f^{n}=\mathrm{Id}\right](\{w\}) \leq\left[f^{p}=\mathrm{Id}\right](\{w\})$ under the characteristic 0 assumption. 
Hence $\sup _{n \in \mathbb{N}}\left(\sup _{w \in \operatorname{supp}\left[f^{n}=\mathrm{Id}\right]}\left[f^{n}=\mathrm{Id}\right](\{w\})\right)<\infty$, so that $\left(\left[f^{n}=\mathrm{Id}\right] \times\left[f^{n}=\mathrm{Id}\right]\right)\left(\operatorname{diag}_{\mathbb{P}^{1}(\bar{k})}\right) \leq\left(d^{n}+1\right) \cdot \sup _{w \in \operatorname{supp}\left[f^{n}=\mathrm{Id}\right]}\left[f^{n}=\mathrm{Id}\right](\{w\})=O\left(d^{n}\right)$ as $n \rightarrow \infty$. Now (1-5) follows from (1-4).

\section{Proof of Theorem 7}

Let $k$ be a field and $k_{s}$ the separable closure of $k$ in $\bar{k}$. Let $p(z) \in k[z]$ be a polynomial of degree $>0$ and $\left\{z_{1}, \ldots, z_{m}\right\}$ the set of all distinct zeros of $p(z)$ in $\vec{k}$ so that $p(z)=a \cdot \prod_{j=1}^{m}\left(z-z_{j}\right)^{d_{j}}$ in $\bar{k}[z]$ for some $a \in k \backslash\{0\}$ and some sequence $\left(d_{j}\right)_{j=1}^{m}$ in $\mathbb{N}$. For a while, we do not assume $\left\{z_{1}, \ldots, z_{m}\right\} \subset k_{s}$. Let $\left\{p_{1}(z), p_{2}(z), \ldots, p_{N}(z)\right\}$ be the set of all mutually distinct, nonconstant, irreducible, and monic factors of $p(z)$ in $k[z]$, so that $p(z)=a \cdot \prod_{\ell=1}^{N} p_{\ell}(z)^{s_{\ell}}$ in $k[z]$ for some sequence $\left(s_{\ell}\right)_{\ell=1}^{N}$ in $\mathbb{N}$. For every $\ell \in\{1,2, \ldots, N\}$, by the irreducibility of $p_{\ell}(z)$ in $k[z], p_{\ell}(z)$ is the unique monic minimal polynomial in $k[z]$ of each zero of $p_{\ell}(z)$ in $\vec{k}$, so $p_{\ell}(z)$ and $p_{n}(z)$ have no common zeros in $\bar{k}$ if $\ell \neq n$. Hence for each $j \in\{1,2, \ldots, m\}$, there is a unique $\ell=: \ell(j) \in\{1,2, \ldots, N\}$ such that $p_{\ell}\left(z_{j}\right)=0$.

Now suppose that $\left\{z_{1}, z_{2}, \ldots, z_{m}\right\} \subset k_{s}$. Then for every $\ell \in\{1,2, \ldots, N\}$, $p_{\ell}(z)=\prod_{i: \ell(i)=\ell}\left(z-z_{i}\right)$ in $\bar{k}[z]$, so that

$$
d_{i}=s_{\ell(i)}
$$

for every $i \in\{1,2, \ldots, m\}$. For every distinct $\ell, n \in\{1,2, \ldots, N\}$,

$$
\prod_{j: \ell(j)=\ell} \prod_{i: \ell(i)=n}\left(z_{j}-z_{i}\right)=\prod_{j: \ell(j)=\ell} p_{n}\left(z_{j}\right)=R\left(p_{\ell}, p_{n}\right)
$$

where $R(p, q) \in k$ is the (usual) resultant of $p(z), q(z) \in k[z]$. The derivation $p_{\ell}^{\prime}(z)$ of $p_{\ell}(z)$ in $k[z]$ satisfies

$$
p_{\ell}^{\prime}(z)=\sum_{h: \ell(h)=\ell}\left(\prod_{\substack{i: i \neq h, \ell(i)=\ell}}\left(z-z_{i}\right)\right)
$$

in $\bar{k}[z]$. Hence for every $\ell \in\{1,2, \ldots, N\}$,

$$
\prod_{j: \ell(j)=\ell} \prod_{\substack{i: i \neq j, \ell(i)=\ell}}\left(z_{j}-z_{i}\right)=\prod_{j: \ell(j)=\ell} p_{\ell}^{\prime}\left(z_{j}\right)=R\left(p_{\ell}, p_{\ell}^{\prime}\right) .
$$

By (11-1), (11-3), and (11-2), we have 


$$
\begin{aligned}
D^{*}(p) & :=\prod_{j=1}^{m} \prod_{i: i \neq j}\left(z_{j}-z_{i}\right)^{d_{i} d_{j}}=\prod_{j=1}^{m} \prod_{i: i \neq j}\left(z_{j}-z_{i}\right)^{s_{\ell(i)} s_{\ell(j)}} \\
& =\prod_{\ell=1}^{N}\left(\prod_{j: \ell(j)=\ell}\left(\left(\prod_{\substack{i: i \neq j, \ell(i)=\ell}}\left(z_{j}-z_{i}\right)^{s_{\ell}^{2}}\right)\left(\prod_{n: n \neq \ell} \prod_{i: \ell(i)=n}\left(z_{j}-z_{i}\right)^{s_{n} s_{\ell}}\right)\right)\right) \\
& =\prod_{\ell=1}^{N}\left(R\left(p_{\ell}, p_{\ell}^{\prime}\right)^{s_{\ell}^{2}} \cdot \prod_{n: n \neq \ell} R\left(p_{\ell}, p_{n}\right)^{s_{n} s_{\ell}}\right),
\end{aligned}
$$

which is in $k \backslash\{0\}$. Now the proof is complete.

\section{Acknowledgements}

The author thanks the referee for their very careful scrutiny and invaluable comments, and also thanks Professors Mamoru Asada and Joe Silverman for discussions on Theorem 7 and comments. This work was partly done during the author's visits to National Taiwan Normal University and Academia Sinica, and the author thanks the institutes and Professor Liang-Chung Hsia for their hospitality. This work was partially supported by JSPS Grant-in-Aid for Young Scientists (B), 24740087.

\section{References}

[Autissier 2001] P. Autissier, "Points entiers sur les surfaces arithmétiques", J. Reine Angew. Math. 531 (2001), 201-235. MR 2002a:11066 Zbl 1007.11041

[Baker 2009] M. H. Baker, "A finiteness theorem for canonical heights attached to rational maps over function fields", J. Reine Angew. Math. 626 (2009), 205-233. MR 2011c:14075 Zbl 1187.37133

[Baker and Hsia 2005] M. H. Baker and L.-C. Hsia, "Canonical heights, transfinite diameters, and polynomial dynamics”, J. Reine Angew. Math. 585 (2005), 61-92. MR 2006i:11071 Zbl 1071.11040

[Baker and Rumely 2006] M. H. Baker and R. Rumely, "Equidistribution of small points, rational dynamics, and potential theory", Ann. Inst. Fourier (Grenoble) 56:3 (2006), 625-688. MR 2007m:11082 Zbl 1234.11082

[Baker and Rumely 2010] M. H. Baker and R. Rumely, Potential theory and dynamics on the Berkovich projective line, Mathematical Surveys and Monographs 159, American Mathematical Society, Providence, RI, 2010. MR 2012d:37213 Zbl 1196.14002

[Benedetto 2010] R. Benedetto, "Non-Archimedean dynamics in dimension one: lecture notes", lecture notes, 2010, http://math.arizona.edu/ swc/aws/2010/2010BenedettoNotes-09Mar.pdf.

[Berkovich 1990] V. G. Berkovich, Spectral theory and analytic geometry over non-Archimedean fields, Mathematical Surveys and Monographs 33, American Mathematical Society, Providence, RI, 1990. MR 91k:32038 Zbl 0715.14013

[Berman and Boucksom 2010] R. Berman and S. Boucksom, "Growth of balls of holomorphic sections and energy at equilibrium”, Invent. Math. 181:2 (2010), 337-394. MR 2011h:32021 Zbl 1208.32020

[Berman, Boucksom, and Nyström 2011] R. Berman, S. Boucksom, and D. Witt Nyström, "Fekete points and convergence towards equilibrium measures on complex manifolds", Acta Math. 207:1 (2011), 1-27. MR 2012j:32036 Zbl 1241.32030 
[Berteloot and Mayer 2001] F. Berteloot and V. Mayer, Rudiments de dynamique holomorphe, Cours Spécialisés 7, Société Mathématique de France, Paris, 2001. MR 2005b:37087 Zbl 1051.37019

[Bilu 1997] Y. Bilu, "Limit distribution of small points on algebraic tori", Duke Math. J. 89:3 (1997), 465-476. MR 98m:11067 Zbl 0918.11035

[Bosch, Güntzer, and Remmert 1984] S. Bosch, U. Güntzer, and R. Remmert, Non-Archimedean analysis: a systematic approach to rigid analytic geometry, Grundlehren der Mathematischen Wissenschaften 261, Springer, Berlin, 1984. MR 86b:32031 Zbl 0539.14017

[Brjuno 1971] A. D. Brjuno, “Аналитическая форма дифференциальных уравнений, 1”, Trudy Moskov. Mat. Obšč. 25 (1971), 119-262. Translated as "Analytic form of differential equations, 1” in Trans. Mosc. Math. Soc. 25 (1971), 131-288. MR 51 \#13365 Zbl 0263.34003

[Brjuno 1972] A. D. Brjuno, “Аналитическая форма дифференциальных уравнений, 2”, Trudy Moskov. Mat. Obšč. 26 (1972), 199-239. Translated as "Analytic form of differential equations, 2” in Trans. Mosc. Math. Soc. 26 (1972), 199-239. MR 51 \#13365 Zbl 0269.34006

[Brolin 1965] H. Brolin, "Invariant sets under iteration of rational functions", Ark. Mat. 6 (1965), 103-144. MR 33 \#2805 Zbl 0127.03401

[Chambert-Loir 2000] A. Chambert-Loir, "Points de petite hauteur sur les variétés semi-abéliennes", Ann. Sci. École Norm. Sup. (4) 33:6 (2000), 789-821. MR 2002e:14037 Zbl 1018.11034

[Chambert-Loir 2006] A. Chambert-Loir, "Mesures et équidistribution sur les espaces de Berkovich", J. Reine Angew. Math. 595 (2006), 215-235. MR 2008b:14040 Zbl 1112.14022

[Cremer 1928] H. Cremer, “Zum Zentrumproblem”, Math. Ann. 98:1 (1928), 151-163. MR 1512397 JFM 53.0303.04

[DeMarco 2003] L. DeMarco, "Dynamics of rational maps: Lyapunov exponents, bifurcations, and capacity”, Math. Ann. 326:1 (2003), 43-73. MR 2004f:32044 Zbl 1032.37029

[Drasin and Okuyama 2007] D. Drasin and Y. Okuyama, "Equidistribution and Nevanlinna theory", Bull. Lond. Math. Soc. 39:4 (2007), 603-613. MR 2008f:37099 Zbl 1123.37018

[Eklof 1973] P. C. Eklof, "Lefschetz's principle and local functors", Proc. Amer. Math. Soc. 37 (1973), 333-339. MR 48 \#3736 Zbl 0254.14004

[Fatou 1920] P. Fatou, "Sur les équations fonctionnelles", Bull. Soc. Math. France 48 (1920), 208-314. MR 1504797 JFM 47.0921.02

[Favre and Jonsson 2004] C. Favre and M. Jonsson, The valuative tree, Lecture Notes in Mathematics 1853, Springer, Berlin, 2004. MR 2006a:13008 Zbl 1064.14024

[Favre and Rivera-Letelier 2006] C. Favre and J. Rivera-Letelier, "Équidistribution quantitative des points de petite hauteur sur la droite projective", Math. Ann. 335:2 (2006), 311-361. Correction in 339:4 (2007), 799-801. MR 2007g:11074 Zbl 1175.11029

[Favre and Rivera-Letelier 2010] C. Favre and J. Rivera-Letelier, "Théorie ergodique des fractions rationnelles sur un corps ultramétrique”, Proc. Lond. Math. Soc. (3) 100:1 (2010), 116-154. MR 2011b:37190 Zbl 1254.37064

[Fekete 1930a] M. Fekete, "Über den transfiniten Durchmesser ebener Punktmengen, 1", Math. Z. 32:1 (1930), 108-114. MR 1545154 JFM 56.0090.01

[Fekete 1930b] M. Fekete, "Über den transfiniten Durchmesser ebener Punktmengen, 2", Math. Z. 32:1 (1930), 215-221. MR 1545162 JFM 56.0112.02

[Fekete 1933] M. Fekete, "Über den transfiniten Durchmesser ebener Punktmengen, 3", Math. Z. 37:1 (1933), 635-646. MR 1545425 Zbl 0007.40204

[Freire, Lopes, and Mañé 1983] A. Freire, A. Lopes, and R. Mañé, "An invariant measure for rational maps”, Bol. Soc. Brasil. Mat. 14:1 (1983), 45-62. MR 85m:58110b Zbl 0568.58027 
[Herman and Yoccoz 1983] M. Herman and J.-C. Yoccoz, "Generalizations of some theorems of small divisors to non-Archimedean fields", pp. 408-447 in Geometric dynamics (Rio de Janeiro, 1981), edited by J. Palis, Jr., Lecture Notes in Math. 1007, Springer, Berlin, 1983. MR 85i:12012 Zbl 0528.58031

[Jonsson 2015] M. Jonsson, "Dynamics of Berkovich spaces in low dimensions", pp. 205-366 in Berkovich spaces and applications (Santiago de Chile/Paris, 2008/2010), edited by A. Ducros et al., Lecture Notes in Math. 2119, Springer, Cham, 2015. MR 3330767 Zbl 06463429

[Lev and Ortega-Cerdà 2012] N. Lev and J. Ortega-Cerdà, "Equidistribution estimates for Fekete points on complex manifolds", preprint, 2012. arXiv 1210.8059v1

[Levenberg 2010] N. Levenberg, "Weighted pluripotential theory results of Berman-Boucksom", preprint, 2010. arXiv 1010.4035

[Ljubich 1983] M. J. Ljubich, "Entropy properties of rational endomorphisms of the Riemann sphere", Ergodic Theory Dynam. Systems 3:3 (1983), 351-385. MR 85k:58049 Zbl 0537.58035

[Milnor 2006] J. Milnor, Dynamics in one complex variable, 3rd ed., Annals of Mathematics Studies 160, Princeton University Press, 2006. MR 2006g:37070 Zbl 1085.30002

[Okuyama 2010] Y. Okuyama, "Nonlinearity of morphisms in non-Archimedean and complex dynamics”, Michigan Math. J. 59:3 (2010), 505-515. MR 2012d:37208 Zbl 1242.37063

[Okuyama 2013a] Y. Okuyama, "Adelic equidistribution, characterization of equidistribution, and a general equidistribution theorem in non-Archimedean dynamics", Acta Arith. 161:2 (2013), 101-125. MR 3141914 Zbl 1302.37070

[Okuyama 2013b] Y. Okuyama, "Fekete configuration, quantitative equidistribution and wandering critical orbits in non-Archimedean dynamics", Math. Z. 273:3-4 (2013), 811-837. MR 3030679 Zbl 06149057

[Okuyama and Stawiska 2011] Y. Okuyama and M. Stawiska, "Potential theory and a characterization of polynomials in complex dynamics", Conform. Geom. Dyn. 15 (2011), 152-159. MR 2846305 Zbl 1252.37036

[Pérez-Marco 1993] R. Pérez-Marco, "Sur les dynamiques holomorphes non linéarisables et une conjecture de V. I. Arnold”, Ann. Sci. École Norm. Sup. (4) 26:5 (1993), 565-644. MR 95a:58103 Zbl 0812.58051

[Pérez-Marco 2001] R. Pérez-Marco, "Total convergence or general divergence in small divisors", Comm. Math. Phys. 223:3 (2001), 451-464. MR 2003d:37063 Zbl 1161.37331

[Rivera-Letelier 2003] J. Rivera-Letelier, "Dynamique des fonctions rationnelles sur des corps locaux", pp. 147-230 in Geometric methods in dynamics, II (Rio de Janeiro, 2000), edited by W. de Melo et al., Astérisque 287, Société Mathématique de France, Paris, 2003. MR 2005f:37100 Zbl 1140.37336

[Rumely 1999] R. Rumely, “On Bilu's equidistribution theorem”, pp. 159-166 in Spectral problems in geometry and arithmetic (Iowa City, IA, 1997), edited by T. Branson, Contemp. Math. 237, American Mathematical Society, Providence, RI, 1999. MR 2000g:11060 Zbl 1029.11030

[Saff and Totik 1997] E. B. Saff and V. Totik, Logarithmic potentials with external fields, Grundlehren der Mathematischen Wissenschaften 316, Springer, Berlin, 1997. MR 99h:31001 Zbl 0881.31001

[Siegel 1942] C. L. Siegel, "Iteration of analytic functions", Ann. of Math. (2) 43 (1942), 607-612. MR 4,76c Zbl 0061.14904

[Silverman 2007] J. H. Silverman, The arithmetic of dynamical systems, Graduate Texts in Mathematics 241, Springer, New York, 2007. MR 2008c:11002 Zbl 1130.37001

[Szpiro, Ullmo, and Zhang 1997] L. Szpiro, E. Ullmo, and S. Zhang, "Équirépartition des petits points”, Invent. Math. 127:2 (1997), 337-347. MR 98i:14027 Zbl 0991.11035 
[Thuillier 2005] A. Thuillier, Théorie du potentiel sur les courbes en géométrie analytique non Archimédienne: applications à la théorie d'Arakelov, thesis, Université Rennes 1, 2005, https:// tel.archives-ouvertes.fr/tel-00010990.

[Tsuji 1959] M. Tsuji, Potential theory in modern function theory, Maruzen, Tokyo, 1959. Reprinted by Chelsea, New York, 1975. MR 22 \#5712 Zbl 0087.28401

[Villani 2009] C. Villani, Optimal transport: old and new, Grundlehren der Mathematischen Wissenschaften 338, Springer, Berlin, 2009. MR 2010f:49001 Zbl 1156.53003

[Yoccoz 1988] J.-C. Yoccoz, "Linéarisation des germes de difféomorphismes holomorphes de (C, 0)", C. R. Acad. Sci. Paris Sér. I Math. 306:1 (1988), 55-58. MR 89i:58123 Zbl 0668.58010

[Yoccoz 1995] J.-C. Yoccoz, "Théorème de Siegel, nombres de Bruno et polynômes quadratiques", pp. 3-88 in Petits diviseurs en dimension 1, Astérisque 231, Société Mathématique de France, Paris, 1995. MR 96m:58214 Zbl 0836.30001

[Yuan 2008] X. Yuan, "Big line bundles over arithmetic varieties", Invent. Math. 173:3 (2008), 603-649. MR 2010b:14049 Zbl 1146.14016

Received July 29, 2014. Revised April 5, 2015.

YÛSUKE OKUYAMA

DIVISION OF MATHEMATICS

Kyoto Institute of TeChNology

SAKYO-KU, KYOTO 606-8585

JAPAN

okuyama@kit.ac.jp 


\title{
PACIFIC JOURNAL OF MATHEMATICS
}

\author{
msp.org/pjm
}

Founded in 1951 by E. F. Beckenbach (1906-1982) and F. Wolf (1904-1989)

\section{EDITORS}

Don Blasius (Managing Editor)

Department of Mathematics

University of California

Los Angeles, CA 90095-1555

blasius@math.ucla.edu

\author{
Paul Balmer \\ Department of Mathematics \\ University of California \\ Los Angeles, CA 90095-1555 \\ balmer@math.ucla.edu \\ Robert Finn \\ Department of Mathematics \\ Stanford University \\ Stanford, CA 94305-2125 \\ finn@math.stanford.edu \\ Sorin Popa \\ Department of Mathematics \\ University of California \\ Los Angeles, CA 90095-1555 \\ popa@math.ucla.edu
}

\author{
Vyjayanthi Chari \\ Department of Mathematics \\ University of California \\ Riverside, CA 92521-0135 \\ chari@math.ucr.edu \\ Kefeng Liu \\ Department of Mathematics \\ University of California \\ Los Angeles, CA 90095-1555 \\ liu@math.ucla.edu \\ Jie Qing \\ Department of Mathematics \\ University of California \\ Santa Cruz, CA 95064 \\ qing@ cats.ucsc.edu
}

\section{PRODUCTION}

Silvio Levy, Scientific Editor, production@msp.org

\section{SUPPORTING INSTITUTIONS}

ACADEMIA SINICA, TAIPEI

CALIFORNIA INST. OF TECHNOLOGY

INST. DE MATEMÁTICA PURA E APLICADA

KEIO UNIVERSITY

MATH. SCIENCES RESEARCH INSTITUTE

NEW MEXICO STATE UNIV.

OREGON STATE UNIV.

\author{
STANFORD UNIVERSITY \\ UNIV. OF BRITISH COLUMBIA \\ UNIV. OF CALIFORNIA, BERKELEY \\ UNIV. OF CALIFORNIA, DAVIS \\ UNIV. OF CALIFORNIA, LOS ANGELES \\ UNIV. OF CALIFORNIA, RIVERSIDE \\ UNIV. OF CALIFORNIA, SAN DIEGO \\ UNIV. OF CALIF., SANTA BARBARA
}

\author{
Daryl Cooper \\ Department of Mathematics \\ University of California \\ Santa Barbara, CA 93106-3080 \\ cooper@math.ucsb.edu \\ Jiang-Hua Lu \\ Department of Mathematics \\ The University of Hong Kong \\ Pokfulam Rd., Hong Kong \\ jhlu@maths.hku.hk \\ Paul Yang \\ Department of Mathematics \\ Princeton University \\ Princeton NJ 08544-1000 \\ yang@math.princeton.edu
}

These supporting institutions contribute to the cost of publication of this Journal, but they are not owners or publishers and have no responsibility for its contents or policies.

See inside back cover or msp.org/pjm for submission instructions.

The subscription price for 2016 is US $\$ 440 /$ year for the electronic version, and $\$ 600 /$ year for print and electronic.

Subscriptions, requests for back issues and changes of subscribers address should be sent to Pacific Journal of Mathematics, P.O. Box 4163, Berkeley, CA 94704-0163, U.S.A. The Pacific Journal of Mathematics is indexed by Mathematical Reviews, Zentralblatt MATH, PASCAL CNRS Index, Referativnyi Zhurnal, Current Mathematical Publications and Web of Knowledge (Science Citation Index).

The Pacific Journal of Mathematics (ISSN 0030-8730) at the University of California, c/o Department of Mathematics, 798 Evans Hall \#3840, Berkeley, CA 94720-3840, is published twelve times a year. Periodical rate postage paid at Berkeley, CA 94704, and additional mailing offices. POSTMASTER: send address changes to Pacific Journal of Mathematics, P.O. Box 4163, Berkeley, CA 94704-0163.

PJM peer review and production are managed by EditFLOW ${ }^{\circledR}$ from Mathematical Sciences Publishers.

\section{PUBLISHED BY}

\section{mathematical sciences publishers \\ nonprofit scientific publishing}

http://msp.org/

(C) 2016 Mathematical Sciences Publishers 


\section{PACIFIC JOURNAL OF MATHEMATICS}

Volume $280 \quad$ No. $1 \quad$ January 2016

Stable capillary hypersurfaces in a wedge

JAIGYOUNG CHOE and MIYUKI KoISO

The Chern-Simons invariants for the double of a compression body

DAVID L. DUNCAN

Compactness and the Palais-Smale property for critical Kirchhoff equations in

closed manifolds

EMMANUEL HeBEy

On the equivalence of the definitions of volume of representations

SUNGWOON KIM

Strongly positive representations of even GSpin groups

YEANSU KIM

An Orlik-Raymond type classification of simply connected 6-dimensional torus manifolds with vanishing odd-degree cohomology

\section{SHINTARÔ KUROKI}

Solutions with large number of peaks for the supercritical Hénon equation

Zhongyuan LiU and ShuangJie Peng

Effective divisors on the projective line having small diagonals and small heights and 141 their application to adelic dynamics

YÛSUKE OKUYAMA

Computing higher Frobenius-Schur indicators in fusion categories constructed from inclusions of finite groups

\section{Peter Schauenburg}

Chordal generators and the hydrodynamic normalization for the unit ball

SEBASTIAN SCHLEISSINGER

On a question of A. Balog

ILYA D. SHKREDOV

Uniqueness result on nonnegative solutions of a large class of differential inequalities 241 on Riemannian manifolds

YUHUA SUN

Correction to "Closed orbits of a charge in a weakly exact magnetic field"

WILL J. MERRY 